\title{
Ionospheric Investigations Using the Sweep-Frequency Pulse Technique at Oblique Incidence
}

\author{
Vaughn Agy and Kenneth Davies
}

\begin{abstract}
(March 12, 1959)
This paper describes the present state of oblique-incidence investigations of the ionosphere, using the sweep-frequency pulse technique, with special reference to the work carried out at the National Bureau of Standards. After a short review of the published literature, oblique-incidence sweeps are presented showing the diurnal and seasonal variations on two east-west paths of lengths 1,150 kilometers and 2,370 kilometers. The discrepancies between observed and calculated maximum usable frequencies are presented for both paths and then various phenomena of interest are shown. Finally, the above phenomena are discussed in the light of existing knowledge and theory and, in particular, it is shown that the discrepancies between observed and calculated maximum usable frequencies are unlikely to be caused by magnetoionic deviation of the ray.
\end{abstract}

\section{Introduction}

The sweep-frequency pulse technique for studying the ionosphere is of vital importance in determining the correct application of vertical-incidence ionospheric data to radio communication problems. For a clear understanding of the important factors which govern the operation of high-frequency radio circuits (signal strength, fading, etc.) direct observations of ionospheric propagation are essential.

With the above problems in mind oblique-incidence observations have been made in several countries. The earliest experiments using the sweep-frequency pulse technique appear to be those of Farmer and Ratcliffe [1] ${ }^{1}$ Farmer, Childs, and Cowie [2], and those of Eckersley et al. [3] prior to World War II. During the war Beynon [4] extended these experiments to longer path lengths with improvements in the recording technique. After World War II oblique-incidence work was extended to still greater distances in Germany [5, 6, 7, 8, 9], Canada [10, 11, 12], the United States [13, 14, 15], and Japan [16]. The results of these investigations will be considered in the following section. In the present paper we shall survey the work done in this field with special reference to the experiments carried out by the Bureau.

\section{Survey of Previous Work}

In the earliest experimental work carried out by Farmer and Ratcliffe [1], pulses were transmitted on an approximately north-south path over a distance of $464 \mathrm{~km}$. Farmer, Childs, and Cowie [2] improved the technique by shortening the sweep time and found that, for ionospherically quiet days, there was good agreement between the observed maximum frequencies for the $F 2$-layer and those calculated from a knowledge of the ionosphere overhead at the two ends of the circuit. These observations were con-

\footnotetext{
1 Figures in brackets indicate the literature references at the end of this paper.
}

firmed by Eckersley and others [3]. It was found that the average discrepancy between the observed and calculated maximum frequencies was about 0.5 percent. Beynon's observations [4] on a north-south path $715 \mathrm{~km}$ long showed that the mean $F 2$ maximum usable frequency (MUF) was in excellent agreement with that calculated from end-point vertical-incidence data. Beynon found that the mean of 110 daytime measurements of the MUF factor (MUF/ $f_{0} F 2$ ) diverged by only 0.2 percent from that calculated from vertical-incidence data using the parabolic layer approximation. In the case of the $E$ layer maximum frequencies, no close relationship with the vertical-incidence data was found, probably because of sporadic- $E$ reflections. Indeed, weak $E s$ reflections were found to be almost always present.

A more comprehensive series of oblique-incidence observations was carried out in Europe by Dieminger $[5,6,7,8,9]$ and his coworkers. They made observations over north-south paths of lengths $1,300 \mathrm{~km}$ (Helsinki to Lindau) and 1,900 km (Sodankylä to Lindau) and have found that, in general, the agreement between the observed sweeps and those calculated from vertical-incidence soundings made near the midpoint was good except near the junction point of the high-angle and low-angle traces (MUF) [7]. An "inverted" transmission curve was used to derive the ordinary ray critical frequency from the oblique-incidence sweeps. The derived critical frequencies for both the $F 1$ - and F2-layers were about 1 percent higher than those observed. On the other hand, the median observed MUF's were, on the average, about 4 percent higher than the calculated median value. Echo traces extending from the junction point towards higher frequencies were frequently observed for the $1,300-\mathrm{km}$ path and more frequently for the longer path. The top frequencies of these extensions were up to 10 percent higher than the respective MUF's. No correlation was found between these extensions and sporadic E. Dieminger and Möller [6] found that, under certain conditions, the high-angle ray was present 
over a relatively large frequency range. Furthermore, they showed that during summer midday, at low sunspot activity, the $E$-layer controlled the MUF for a distance of $1,300 \mathrm{~km}$ whereas at vertical incidence the highest frequency was reflected from the F2-layer.

The Canadian experiments $[10,11]$ made over an east-west path of $2,350 \mathrm{~km}$ showed that under certain conditions the Pedersen ray was relatively important and that signals were sometimes received at frequencies up to 10 percent higher than the so-called "classical MUF". In these experiments, good agreement was found between the observed classical MUF's and the MUF's calculated on the basis of Sellmeyer theory. No departures from reciprocity were observed, and it was shown that the frequency separation between the ordinary and the extraordinary traces at the MUF was about 0.3 Mc.

Records showing the importance of the one-hop Pedersen ray in the determination of the MUF were obtained by Warren and Hagg [12] on a 5,300$\mathrm{km}$ path across the North Atlantic. Over this distance the low-angle ray is cut off by the earth. These observations agreed relatively well with calculations by Kift [17].

In the United States the National Bureau of Standards has conducted oblique-incidence experiments since 1951. These were carried out over two eastwest paths; namely, Sterling, Va. (near W ashington, D.C.) to St. Louis, Mo., $1,150 \mathrm{~km}[13,14]$ and Sterling, Va. to Boulder, Colo., 2,370 km [15]. The records obtained from these experiments have been scaled primarily for MUF's. Wieder [14] has shown that for transmission over $1,150 \mathrm{~km}$ the observed MUF's were sometimes 5 percent higher than the MUF's calculated from midpoint data and the appropriate Smith transmission curve [18].

When the Sterling to Boulder experiment was started, it became apparent that care must be taken to distinguish between two frequencies either of which may, with reason, be called the MUF of a given layer. The first of these, referred to variously as "the junction frequency" (of the high-angle and low-angle rays), or the "nose frequency" (because of the shape of the trace seen in a clear sweepfrequency record), is here called the "classical MUF". It is a quantity determined primarily by the geometry of the ray path between transmitter and receiver and is independent of equipment sensitivity or power. The second of the two frequencies is the highest frequency propagated by a given layer. Terms applied to this frequency include both "maximum observed frequency" (MOF) and "practical MUF" for the layer. For the shorter path it is almost always identical with the classical MUF described above but for the longer path it is frequently higher than the classical MUF by as much as 10 percent. When the latter situation holds, the amount by which the maximum observed frequency exceeds the classical MUF is evidently dependent on the power and sensitivity of the equipment used. The difference between the MOF and the classical MUF (expressed either as frequency difference or percentage frequency difference) is referred to as the "MUF extension" or occasionally as the "nose extension". The appearance of the trace on sweep-frequency oblique-incidence records at frequencies above the classical MUF suggests these terms as well as terms like "whisker" and "tail".

A third quantity of importance is the "calculated MUF" ${ }_{2}$ which is the result obtained by application of any one of several methods of computation to the vertical-incidence (midpoint) ionogram (e.g., Appleton-Beynon [19], Smith [20]). When the calculated MUF is derived by means of standard transmission curves of the type devised by Smith, the term "standard MUF" applies. To the extent that theory and experiment agree, the calculated MUF is identical with the classical MUF.

The nose extension was observed only on the longer $(2,370-\mathrm{km})$ path, at least to an appreciable extent. However, Wieder's analysis showed that even in the case of a well-defined MUF (no extension), over the $1,150-\mathrm{km}$ path the average value of the MUF was greater than the value deduced from the vertical-incidence data at the midpoint of the path by between 0 and 10 percent, depending on time of day and season. The mean difference is about 3 percent. On the longer path Sulzer [15] observed echoes on numerous occasions at frequencies considerably above the so-called classical MUF.

The sweep-frequency technique has been used in Japan over distances of about $1,090 \mathrm{~km}$ and about $1,840 \mathrm{~km}$, where it has been found that the mean percent differences between the observed and calculated MUF's for F2-layer propagation is about 3 percent for the $1,090-\mathrm{km}$ path and about 6 percent for the $1,840-\mathrm{km}$ path. Excellent agreement appears to have been found between the observed and calculated regular E-layer MUF's. However, when sporadic $E$ was present, appreciable discrepancies were observed.

Summary of results of previous work:

(1) The existing theories of propagation are adequate for calculating MUF's for distances up to about $700 \mathrm{~km}$. For distances of about $1,000 \mathrm{~km}$, the MUF errors average approximately 3 percent and appear to increase with increasing path length.

(2) MUF extensions are sometimes observed over distances of the order of 1,000 to $2,000 \mathrm{~km}$. For greater distances such extensions are commonly observed.

(3) The high-angle ray is important under certain conditions.

(4) For certain distances the $E$ - and F1-layers determine the MUF, especially in summer daytime during periods of low sunspot activity.

(5) The frequency separation between the ordinary and extraordinary rays at the MUF's decreases with increase of distance from about $0.7 \mathrm{Mc}$ at vertical incidence to about $0.3 \mathrm{Mc}$ at $2,400 \mathrm{~km}$.

2 The IXth Plenary Assembly of the CCIR (Los Angeles, April 1959) adopted a recommendation on the meaning of MUF, advocating the use of the term "classical MUF" to designate the highest frequency transmitted by ionospheric refraction alone. (Our use of the term is consistent with this definition as applied to a particular layer). The recommendation also suggests that "experimental MUF" be used to designate the results of special experiments (our MOF), and
that "theoretical MUF" could be used for the results of theoretical calculations (our "calculated MUF"). 


\section{NBS Oblique-Incidence Program}

The Bureau's oblique-incidence experiment has been in operation since August 1951. From August 1951 to December 1952 observations were made over the $1,150-\mathrm{km}$ path between Sterling, Va., and St. Louis, Mo. A conventional vertical-incidence ionosonde was operated at Batavia, Ohio, the midpoint of the great-circle path. From 1953 onwards, the circuit used was between Sterling, Va., and Boulder, Colo., $(2,370 \mathrm{~km})$ with the midpoint ionosonde located at Carthage, Ill.

The following features will be considered in the

present section: (1) Sample records indicating diurnal and seasonal changes for the two paths; (2) the quantitative relationship between oblique-incidence and vertical-incidence data, including sweepfrequency backscatter data; (3) some interesting phenomena.

\subsection{Sample Sweeps}

In figures 1 and 2 we see the diurnal variations on quiet summer and quiet winter days for the $1,150-\mathrm{km}$ path. It is seen that near noon in summer the MUF is determined by the E-layer and not by the F2layer as is the case at vertical incidence. It should

JUNE 25, 1952
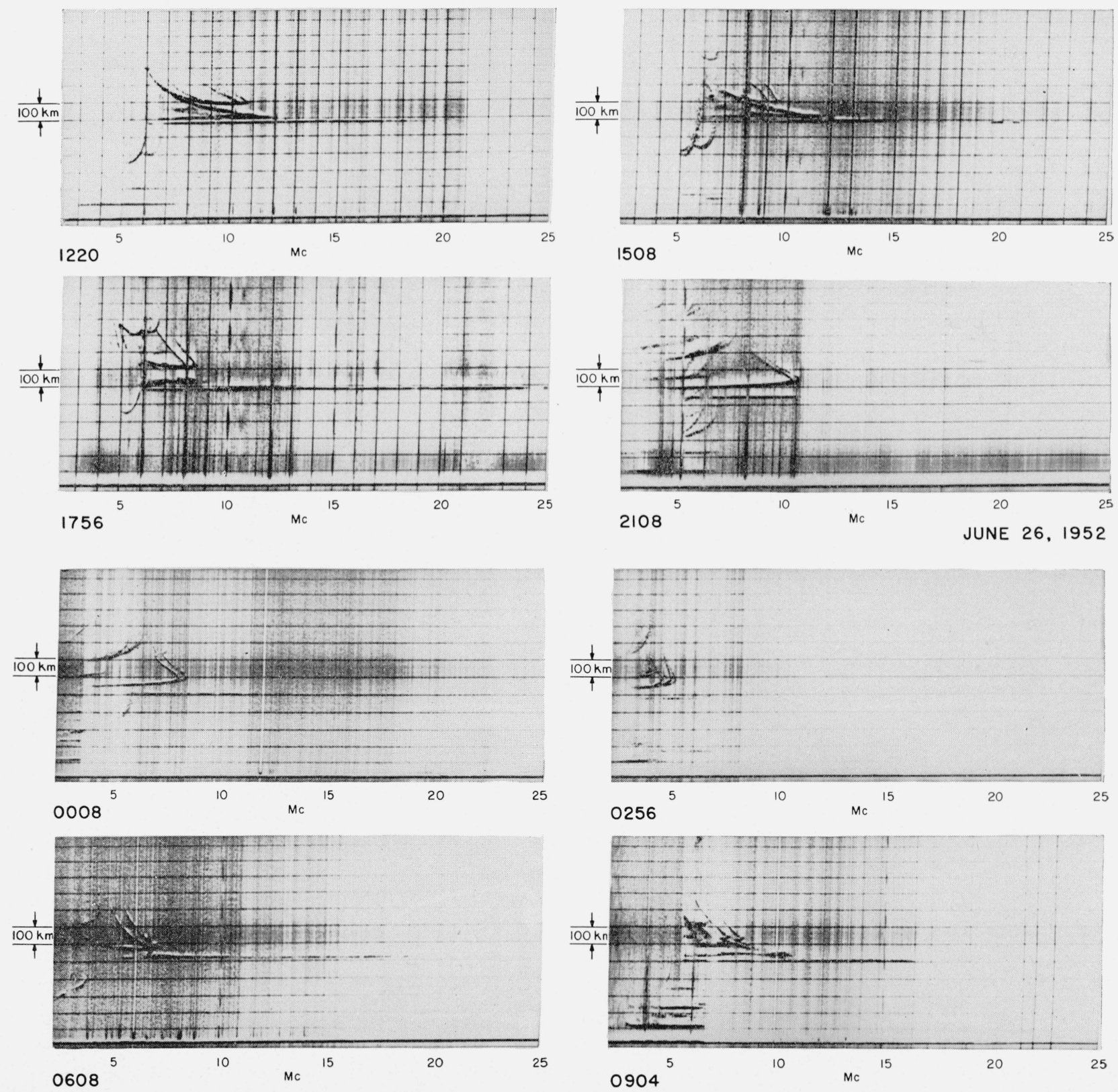

Figure 1. Summer day diurnal sequence for the 1,150-km path, Sterling, Va., to St. Louis, Mo. (June 25, 26, 1952). The virtual path scale, $P^{\prime}$, is $1 / 2(\Delta \mathrm{ct})$ in all sweeps. Times refer to $75^{\circ}$ West Meridian Time. 

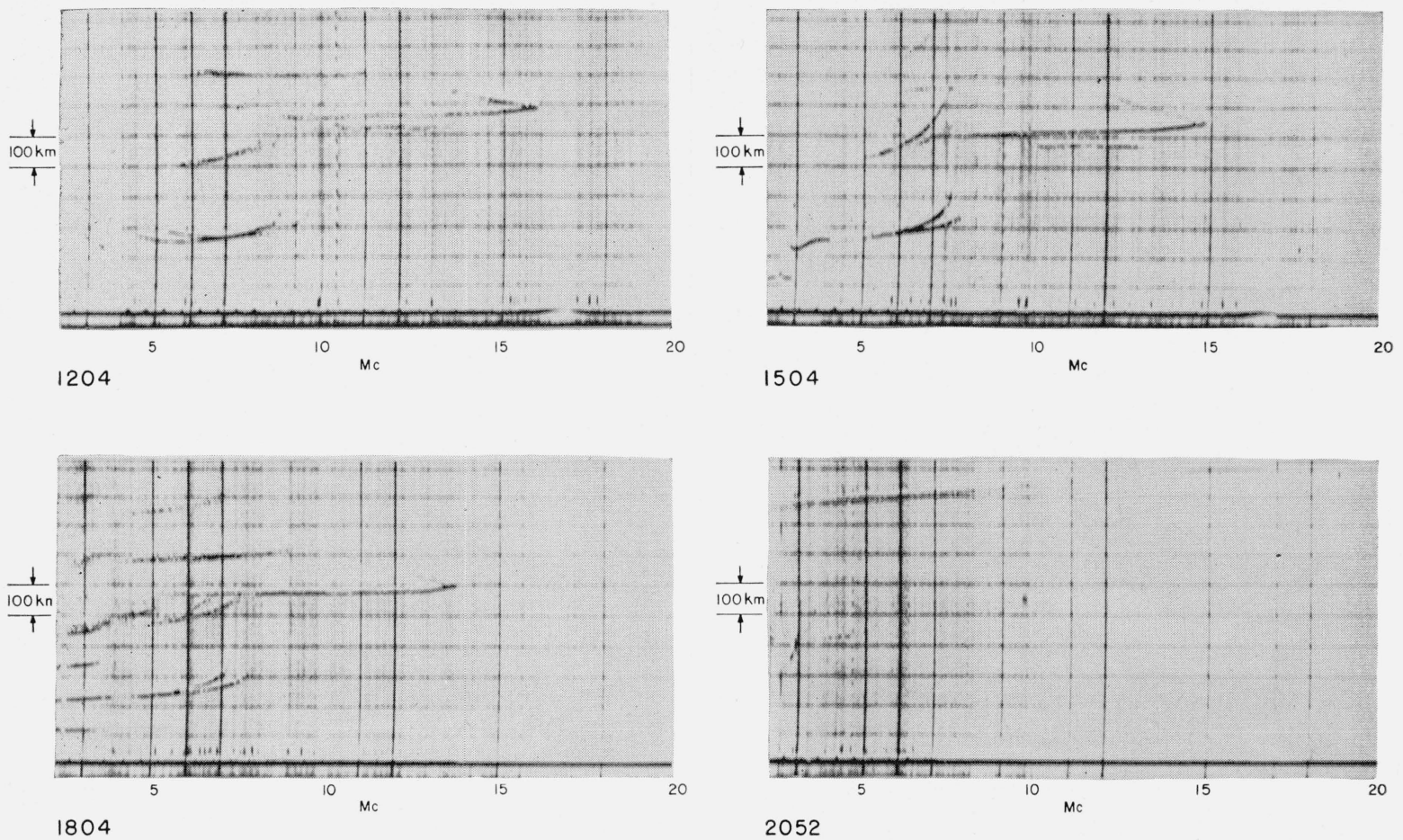

JANUARY 10, 1952
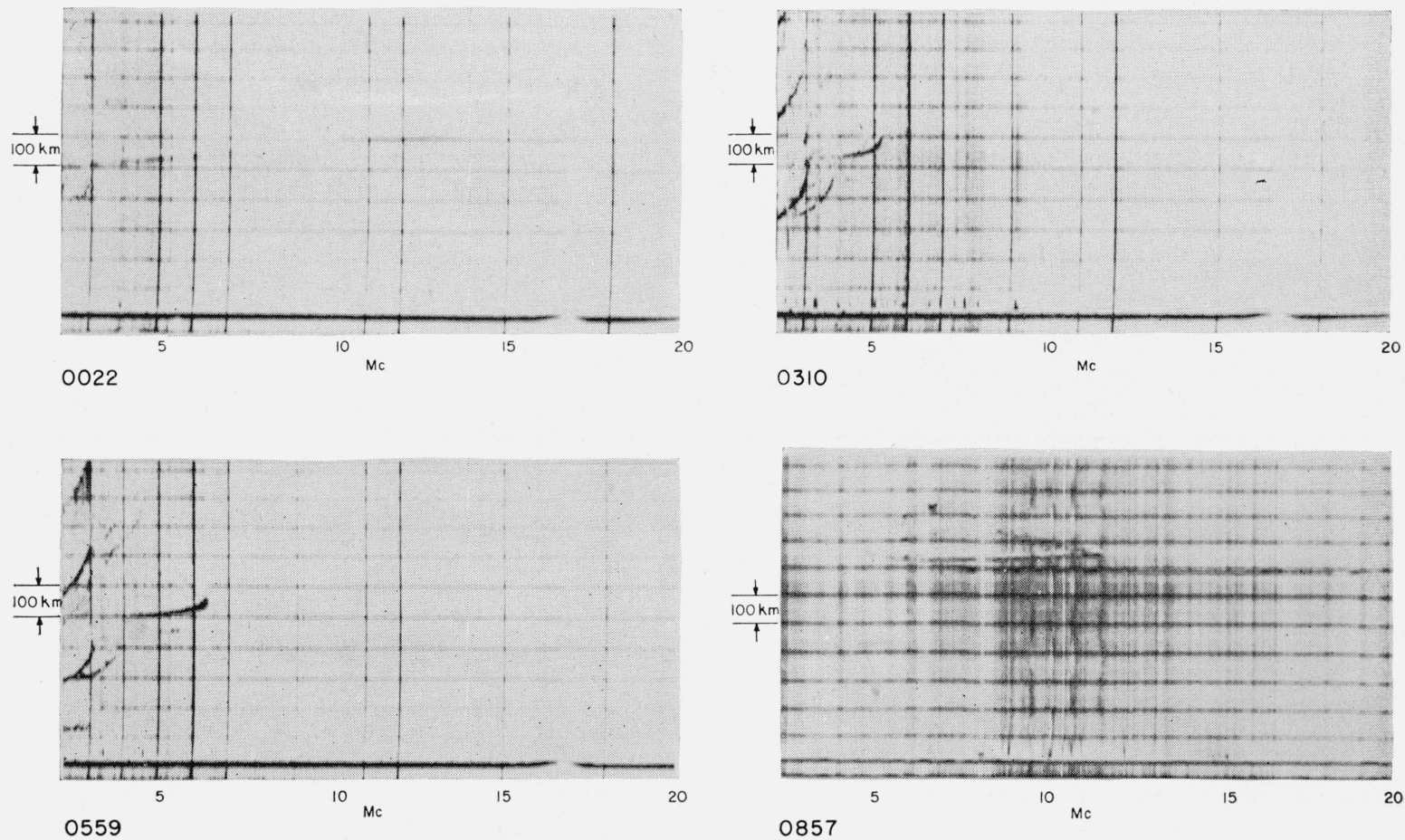

Figure 2. Winter day diurnal sequence for the 1,150-km path, Sterling, Va., to St. Louis, Mo. (Jan. 9, 10, 1952). 
be borne in mind that these observations were made during a period of relatively low sunspot activity. Notice that the F2-layer echo is retarded in the underlying $F$-layer. It will be seen that near noon the F1-layer Pedersen ray is relatively strong down to about $6 \mathrm{Mc}$ at which frequency there is little trace of the low-angle ray, probably because of "cut-off" by Es (see sec. 3.3.a). Near sunset the $F 1$-layer trace disappears, leaving only the $F 2$-layer trace which persists throughout the night. The $F 1$ layer is reformed near sunrise. Sometimes the F1layer is continuous with the night $F 2$-layer, in these cases the daytime $F 2$-layer trace first appears high up on the F1-layer Pedersen ray. On other occasions the F2-layer is continuous with the night $F 2$-layer, and under these conditions the F1-layer trace develops below the low-angle $F 2$ trace. This appears to have been the case on the morning of June 26 , 1952. On a typical winter day (fig. 2) there is little or no evidence of a separate $F 1$ trace. It will also be seen that the Pedersen ray is relatively weak as compared with that for a summer day. This is to be expected on the basis of increased defocusing of the high-angle ray for the thinner wintertime layer. Furthermore, the maximum frequency of the $F$-layer trace occurs near noon in contrast to the case for a summer day, when the highest value is reached near $1800 \mathrm{hr}$. It is seen that on a winter night the F2layer MUF falls to a low value.

Figures 3 and 4 are similar sequences indicating diurnal variations over the $2,370-\mathrm{km}$ path for a summer day and a winter day. Note that during summer midday, say 0900 to 1500 , the $F 1$ layer determines the MUF for the path. Here again, it should be remembered that the period under consideration was one of relatively low sunspot activity. Two-hop Es traces are evident $(2,370 \mathrm{~km}$ is beyond the one-hop limit for reflection heights less than about $110 \mathrm{~km})$; and occasionally the two-hop $E_{\mathrm{s}}$ mode may provide the maximum frequency for the path, particularly during summer daylight hours. Also worth noting is the relative difficulty of specifying precisely the frequency at which the upper and lower $F 1$ traces meet (the classical MUF). The increasing path length with frequency indicated in the first record of the sequence is caused by a drift of the pulse repetition rate of the Sterling equipment relative to that at Boulder; the corresponding record made at Sterling shows a trace with a downward slope. The average ordinate at a given frequency will quite accurately give a measure of the virtual path (delay time) at that frequency if the reciprocity law is valid.

The winter day sequence (fig. 4) shows little if any $F 1$ and the presence of $E_{\mathrm{s}}$ is indicated by the $M$ or $N$ trace lying between those representing the one-hop and two-hop F2 modes, especially on the record for 0603 90th West Meridian Time.

The records for the longer path (Sterling to Boulder) are often more complicated than those for the shorter path. This additional complication shows itself in the number of traces and the some- times confusing relationship among them. More important, from the standpoint of MUF data analysis, is the difficulty of accurate scaling of the MUF produced by the fact that the angle between the upper-ray and lower-ray traces becomes more acute as the path length increases. The finite pulse length combined with the more acute angle may, for the $F 2$ layer, give an uncertainty in the classical MUF of several tenths of a megacycle and for the F1-layer MUF an uncertainty of $1 \mathrm{Mc}$ or more. In those records where it is impossible to define the frequency at which the upper-ray and lower-ray traces meet, the procedure used has been to extrapolate the upper trace along its lower edge (corresponding to the leading edge of the pulse producing it) to intersect with the lower edge of the low-angle trace. Such a procedure provides, if not always accurate values of the classical MUF, at least a degree of consistency which would otherwise be lacking.

\subsection{Vertical and Oblique Incidence Data}

The calculation of MUF's is still one of the most important problems in high-frequency radio communication. Because of its simplicity, the Smith transmission curve [18] has been the practical link between vertical-incidence ionospheric data and oblique-incidence predictions in the Central Radio Propagation Laboratory and many other laboratories. The oblique-incidence experiments described herein have afforded a valuable, although limited, check upon the accuracy of results obtained by its use. In the following paragraphs the discrepancies between the MUF's obtained by the use of the Smith transmission curves and the observed MUF's are considered.

The Smith curves may be defined by the equation $f=k f^{\prime}$ sec $\Phi$ where $f$ is the oblique-incidence frequency, $f^{\prime}$ is the equivalent vertical-incidence frequency, $\Phi$ is the half-angle at the apex of the equivalent path, and $k$ is a correction factor assumed to depend only on transmission distance.

\section{a. Sterling to St. Louis}

Sample winter and summer day plots showing the diurnal variation of observed and calculated MUF are shown in figures 5 and 6 . (All times quoted for the Sterling to St. Louis path refer to the $75^{\circ} \mathrm{West}$ Meridian.) It will be seen that in both cases the measured MUF's are in general higher than those calculated from midpoint records. The average difference between the two MUF's is about 3 percent. It appears that, on the whole, the percent error in the calculated values is greater during the day than during the night. The average daytime monthly error is greater during the winter than during the summer. This is illustrated by the histograms of figure 7. The average error is 3.6 percent for February 1952 and 1.5 percent for May 1952. However, there are many more negative errors in summer so that the spread is much greater. 

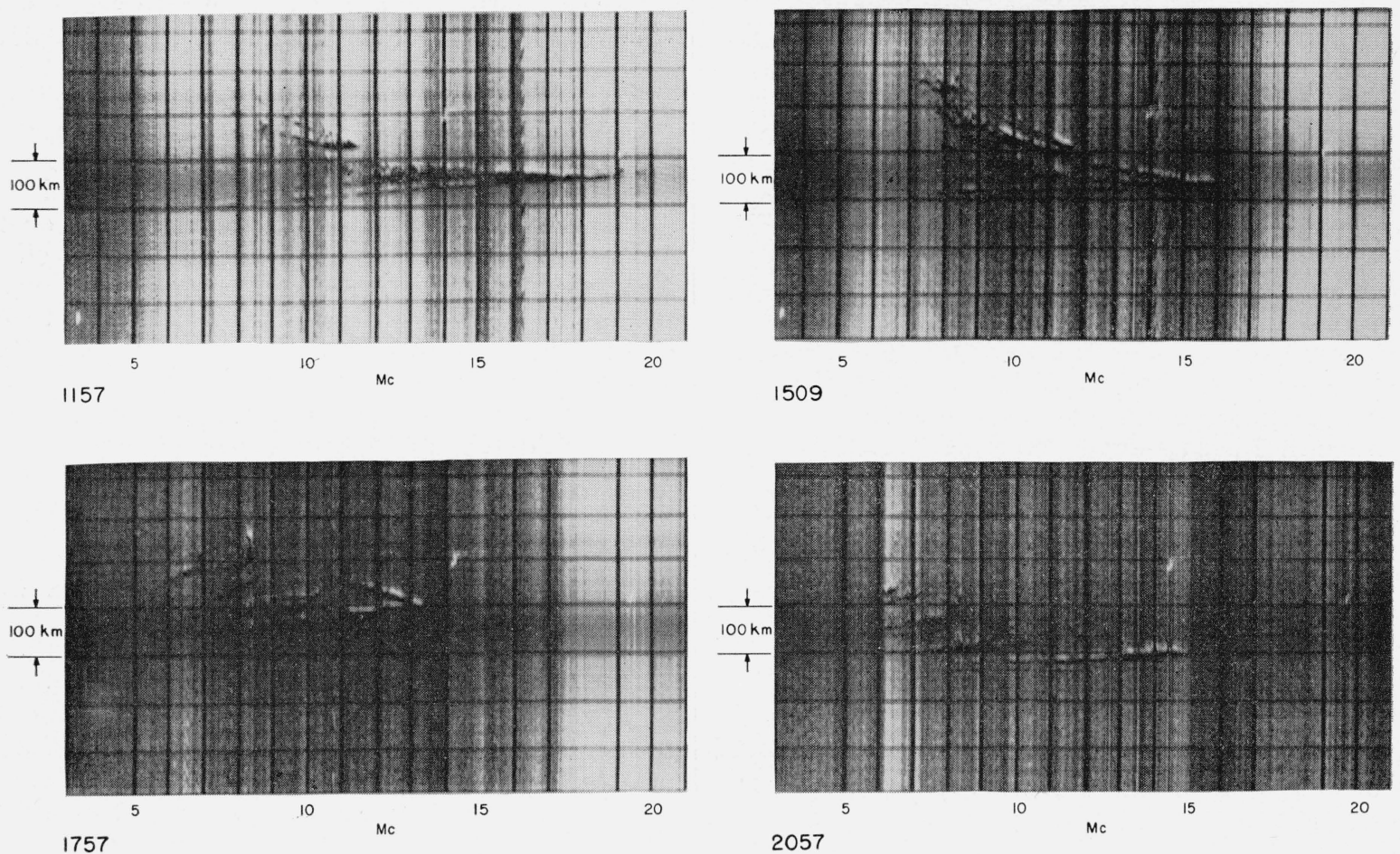

AUGUST $4-5,1954$
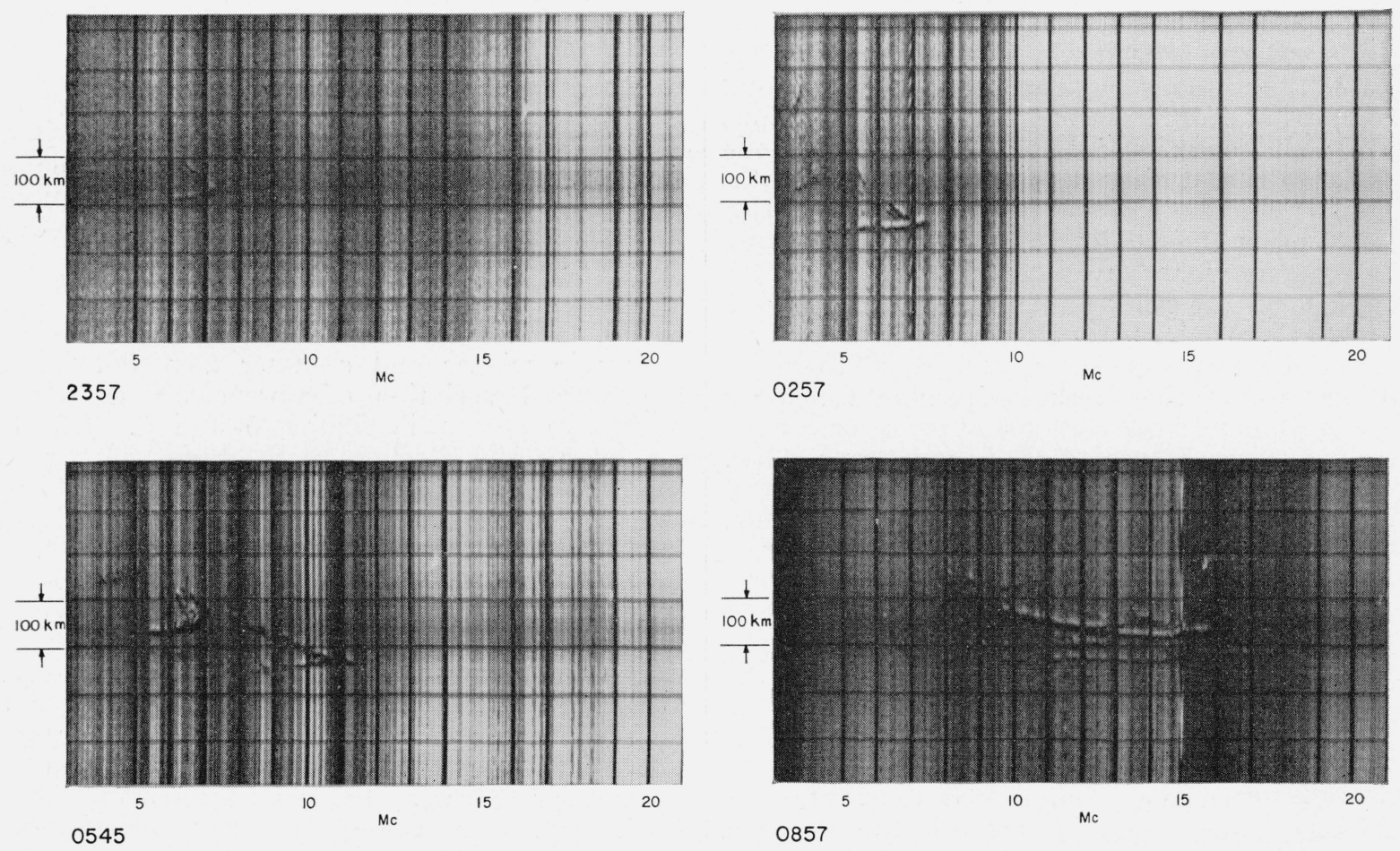

Figure 3. Summer day diurnal sequence for the 2,370-km path, Sterling, Va., to Boulder, Colo. (Aug. 4, 5, 1954). Times refer to $90^{\circ}$ West Meridian Time. 

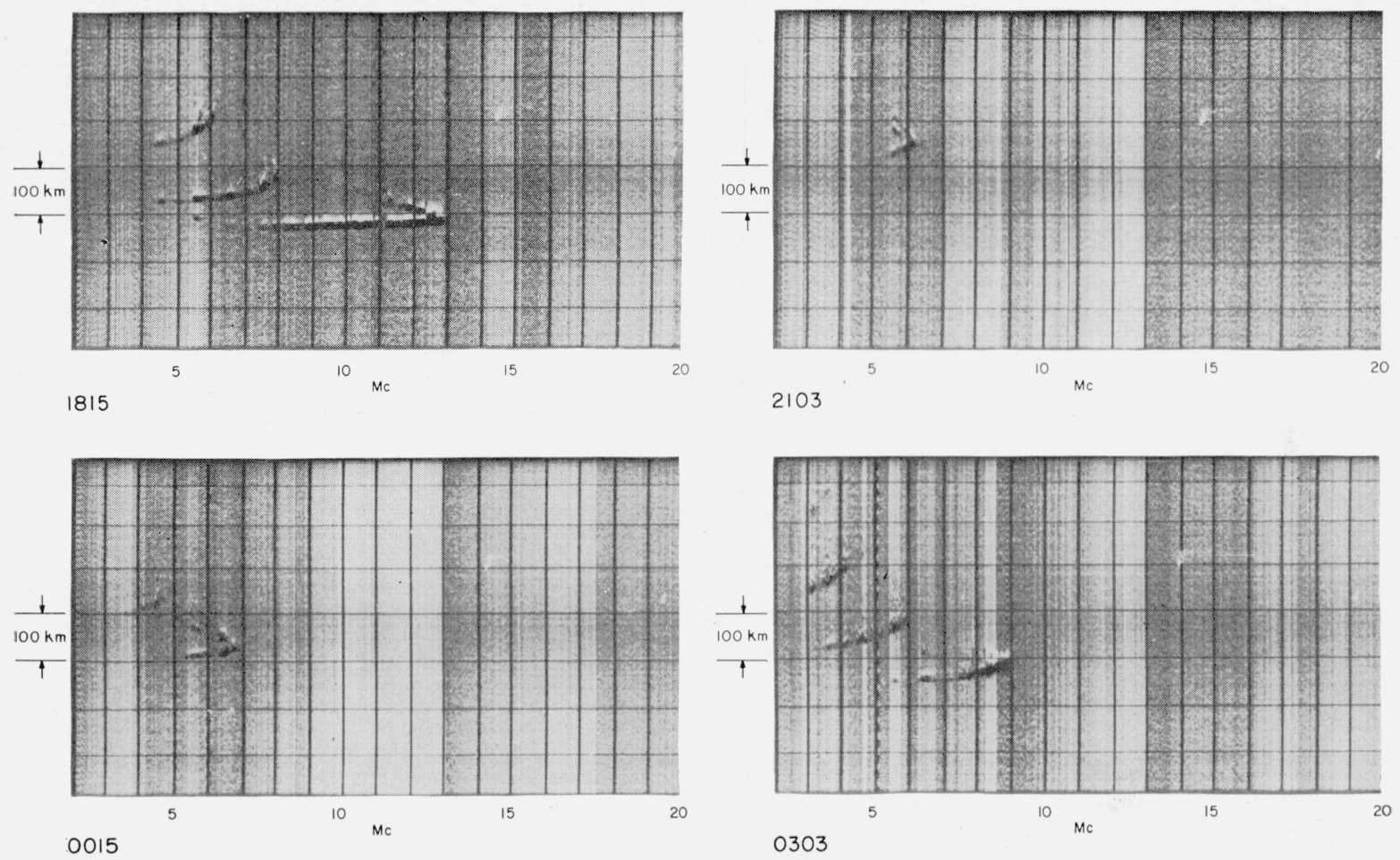

FEBRUARY 4,1954
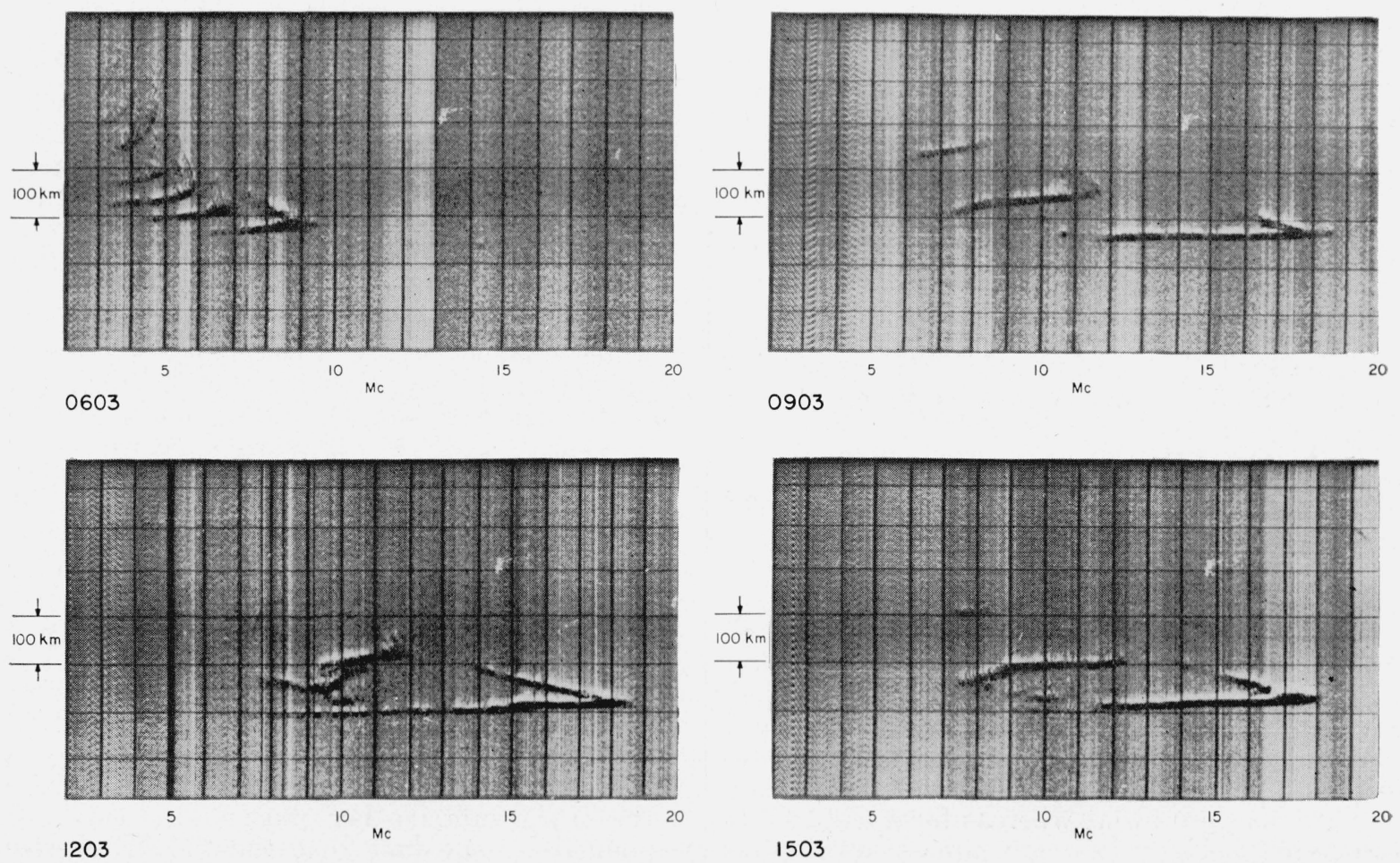

Figure 4. Winter day diurnal sequence for the 2,370-km path, Sterling, Va., to Boulder, Colo. (Feb. 3, 4, 1954). 


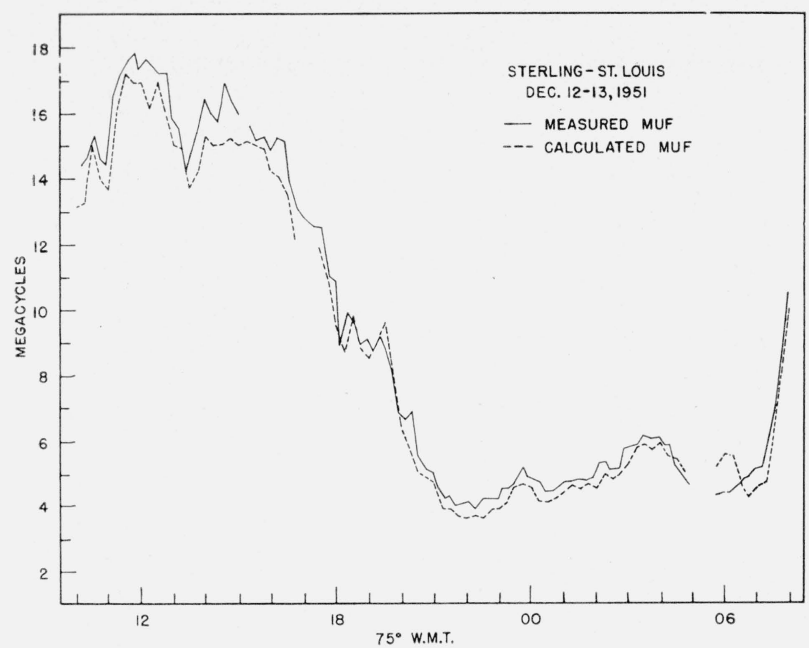

Figure 5. Comparison between the calculated MUF's and the observed MUF's for the 1,150-km path (winter day).

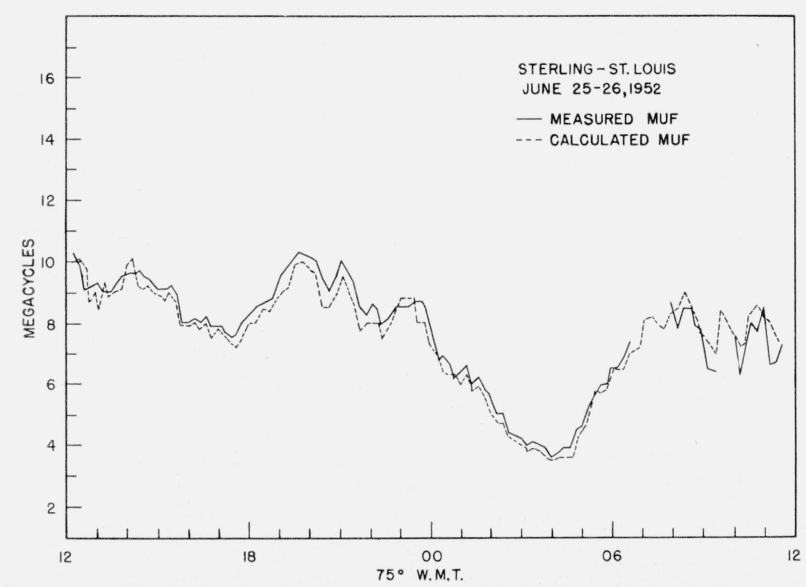

Figure 6. Comparison between the calculated $M U F^{\prime} s$ and the observed MUF's for the 1,150-km path (summer day).

It will be seen that F1-layer MUF's are not included in figures 5 and 6 . These data were excluded because of the uncertainty involved in the determination of the MUF for this layer due to the overlapping of the $F 1$ trace and the two-hop $E$ trace in the vicinity of the F1-layer MUF.

\section{b. Sterling to Boulder}

Figures 8 and 9 are sample plots of the observed and calculated F2-layer MUF's for the 2,370-km path. The magnitude of MUF extensions when present are indicated by the lengths of short vertical lines drawn upward from the plotted points representing the observed classical MUF's. A comparison of these graphs shows that whereas for a winter day the calculated MUF values are almost always less than the observed values, the same is not necessarily true for a summer day. Indeed, near noon in sum-
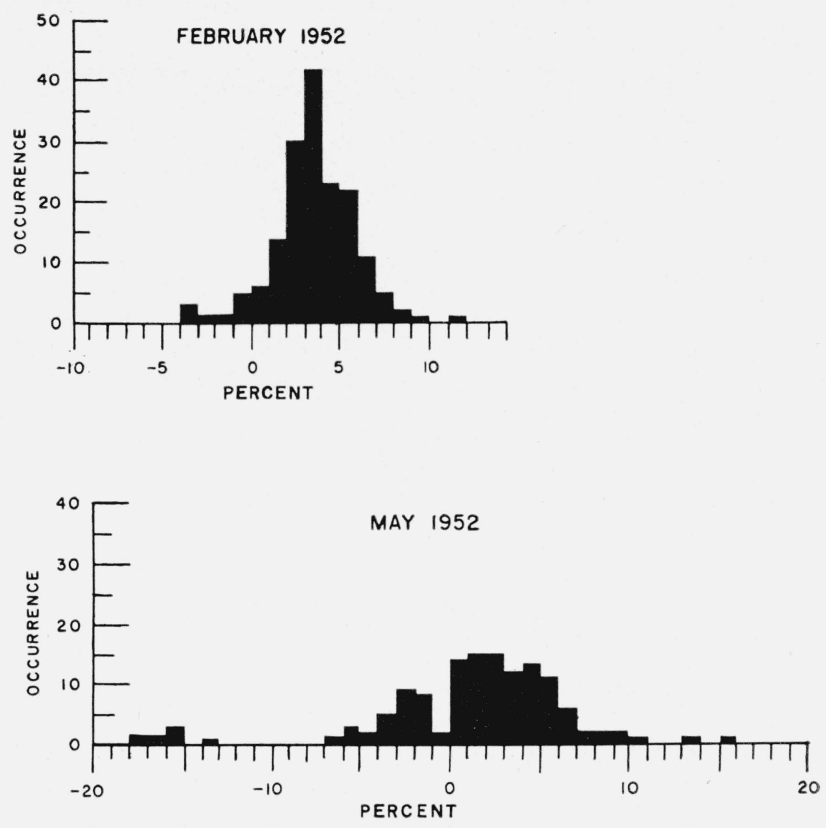

Figure 7. Histograms of 2 month's distributions of the percentage difference between the daytime observed MUF's minus the calculated values for the $1,150-\mathrm{km}$ path, Sterling to St. Louis.

mer the observed MUF may fall well below the calculated value; this is similar to the observations made on the shorter path. It should be noted that at these times the F2-layer echoes are usually heavily retarded in the $F 1$ layer, and the virtual height of reflection is thereby increased. It is likely, as indicated in section 3.4, that the correction factor, $k$, should properly decrease with increasing virtual height. If this adjustment be assumed, the winter to summer differences seen in a comparison of figures 8 and 9 can readily be accounted for. (All times quoted for the Sterling to Boulder path refer to the $90^{\circ}$ West Meridian.)

The histogram in figure 10 presents data from the Sterling to Boulder path. Here, however, the maximum observed frequency for the $F 2$ layer (rather than the classical MUF) has been compared with the calculated MUF. Similarity is seen between figure 10 and figure 7 (Sterling to St. Louis), in that the winter day plot shows somewhat less dispersion than that for the summer day. Whereas, the percentages for the summer day are comparable for the two paths, for the winter day the median percent difference for the longer path is about 7 percent compared with 3 percent for the shorter path. However, the difference in the method of derivation (classical MUF for Sterling to St. Louis, MOF for Sterling to Boulder) appears to be largely responsible for the difference shown (see fig. 18). The agreement between the summer-day plots for the two paths is explained by the fact that the "MUF extension" seldom appears on the $F 2$-layer trace during summer midday even over the longer path. 


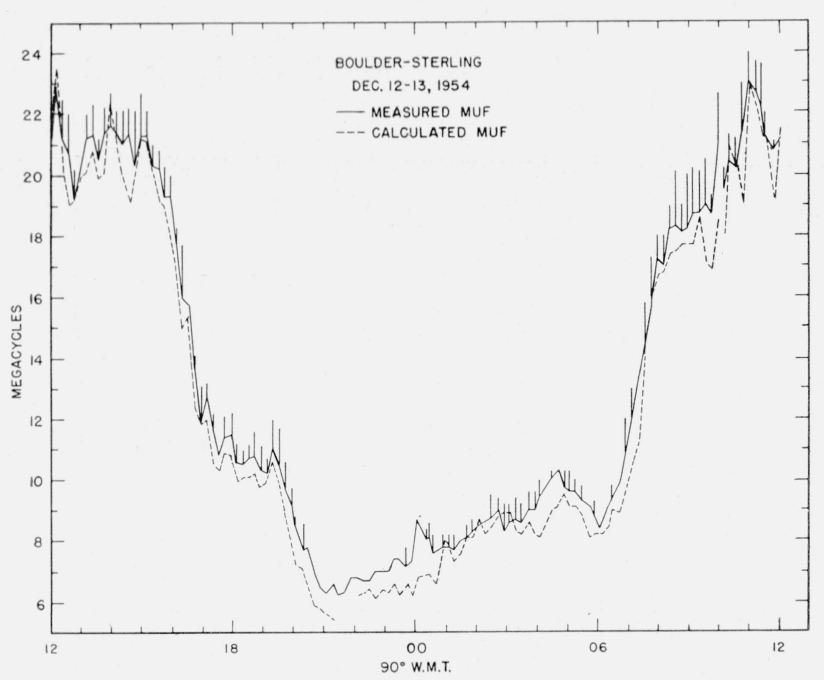

Figure 8. Comparison between the calculated MUF's and the observed MUF's for the 2,370-km path (winter day).

Vertical lines represent MUF extension.

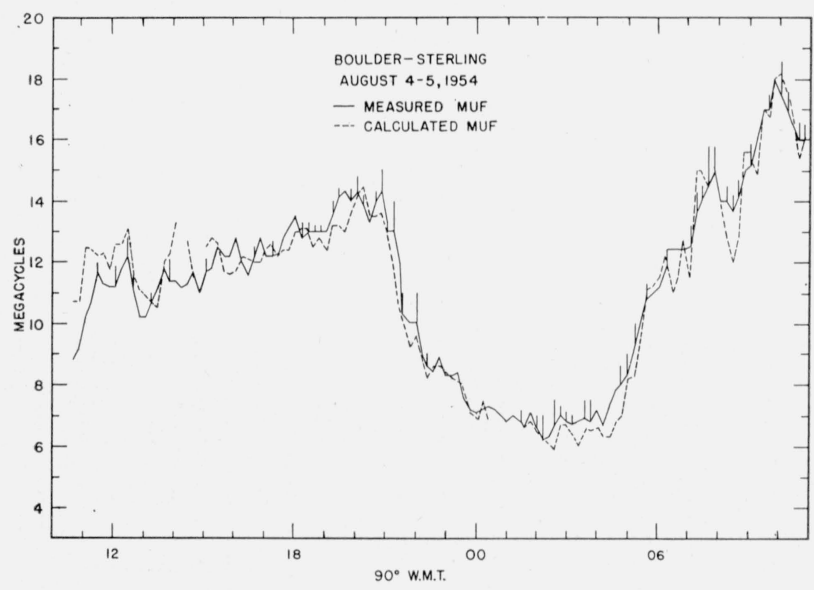

Figure 9. Comparison between the calculated MUF's and the observed MUF's for the 2,370-km path (summer day).

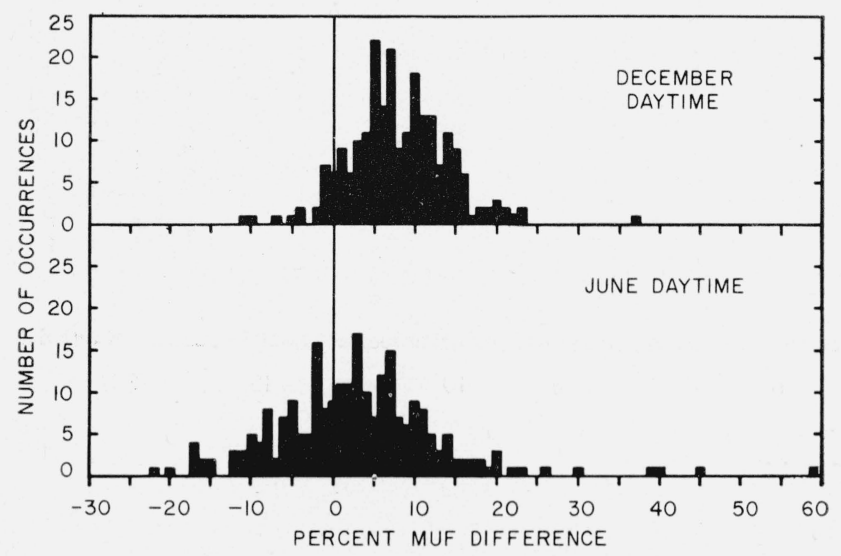

Figure 10. Distribution of percentage difference between the maximum received F2-frequency observed for the Boulder to Sterling path minus the ordinary wave F2 MUF scaled from midpoint records, 1954 .
Another interesting point is brought out in figures $11 \mathrm{a}$ and $11 \mathrm{~b}$. Here the vertical-incidence ionogram (fig. 11b) would probably be scaled as a blackout $(B)$ if it were scaled according to the internationally agreed scaling rules [19]. However, there is a definite echo on the oblique-incidence ionogram. This observation shows that care is needed in applying blackout studies at vertical incidence to communications problems.

\section{c. MUF's Deduced from Sweep-Frequency Backscatter Records}

Maximum usable frequencies can be measured by sweep-frequency pulse observations of backscatter echoes [22]. Backscatter recordings were made at the same time as the oblique-incidence records were made over the Sterling to Boulder path. If we assume that the leading edge of the backscatter pulse is reflected at the edge of the skip distance, then the frequency is the MUF for that distance.

Such a backscatter record is shown in figure 12a along with the $P^{\prime} f$ sweep, figure $12 \mathrm{~b}$, for the Boulder to Sterling path. It will be seen that for an assumed slant range of $2,500 \mathrm{~km}$ the backscatter record yields an MUF of $16.3 \mathrm{Mc}$, which is in good agreement with the value of $16.2 \mathrm{Mc}$ taken from the $P^{\prime} f$ sweep and is slightly larger than the value of $16.0 \mathrm{Mc}$ calculated from the midpoint vertical-incidence data. This result agrees with the observations of Silberstein [23] who found that over this same path the MUF's derived from backscatter data are, on the whole, 0.5 percent greater than those derived from oblique-incidence data. On the other hand, the percent difference between MUF's obtained from vertical-incidence data and those observed at oblique incidence is about -2.0 percent.

\section{d. Spread Echoes}

One of the problems facing the frequency predictor is that of determining the MUF from a spread vertical-incidence record. This problem is of particular importance at high latitudes where even the slightest ionospheric disturbance gives rise to spread echoes. The F2-layer critical frequency normally scaled is generally near the lower frequency limit of the spread F2 trace; and so the question arises whether the normal prediction methods, based on monthly median foF 2 values, are adequate under these conditions.

At the latitudes over which the Bureau experiments were carried out the occurrence of spread echoes is relatively rare and hence a thorough study of this problem has not been possible. However, one example is shown in figures $13 \mathrm{a}$ and $13 \mathrm{~b}$, which shows the oblique-incidence ionogram and the corresponding midpoint vertical-incidence ionogram for the $1,150-\mathrm{km}$ path. Although the oblique-incidence traces show signs of spread, the ordinary ray can be identified from the high-angle ray and the classical MUF is seen to be about 4.1 Mc. The verticalincidence ionogram, on the other hand, shows considerable confusion and it is difficult to decide what 
MAY I, 1952
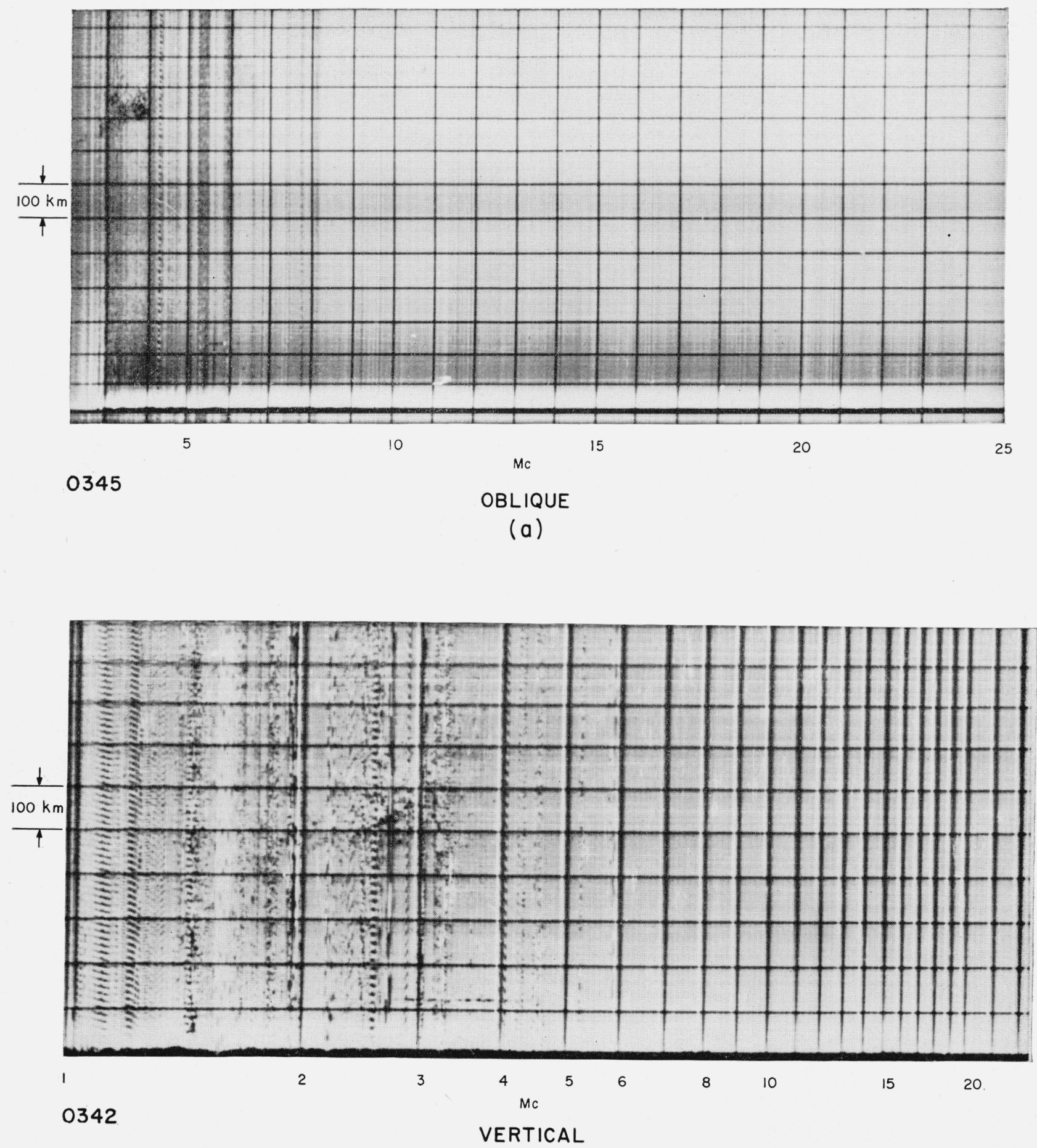

(b)

FIGURE 11. Sweeps showing the presence of echoes on oblique-incidence ionograms (a) and absence on the midpoint vertical-incidence ionograms (b).

Note that the times shown on the oblique-incidence ionograms refer to the end of the sweep which takes 12 min. whereas the vertical-incidence sweep takes 15 sec. 


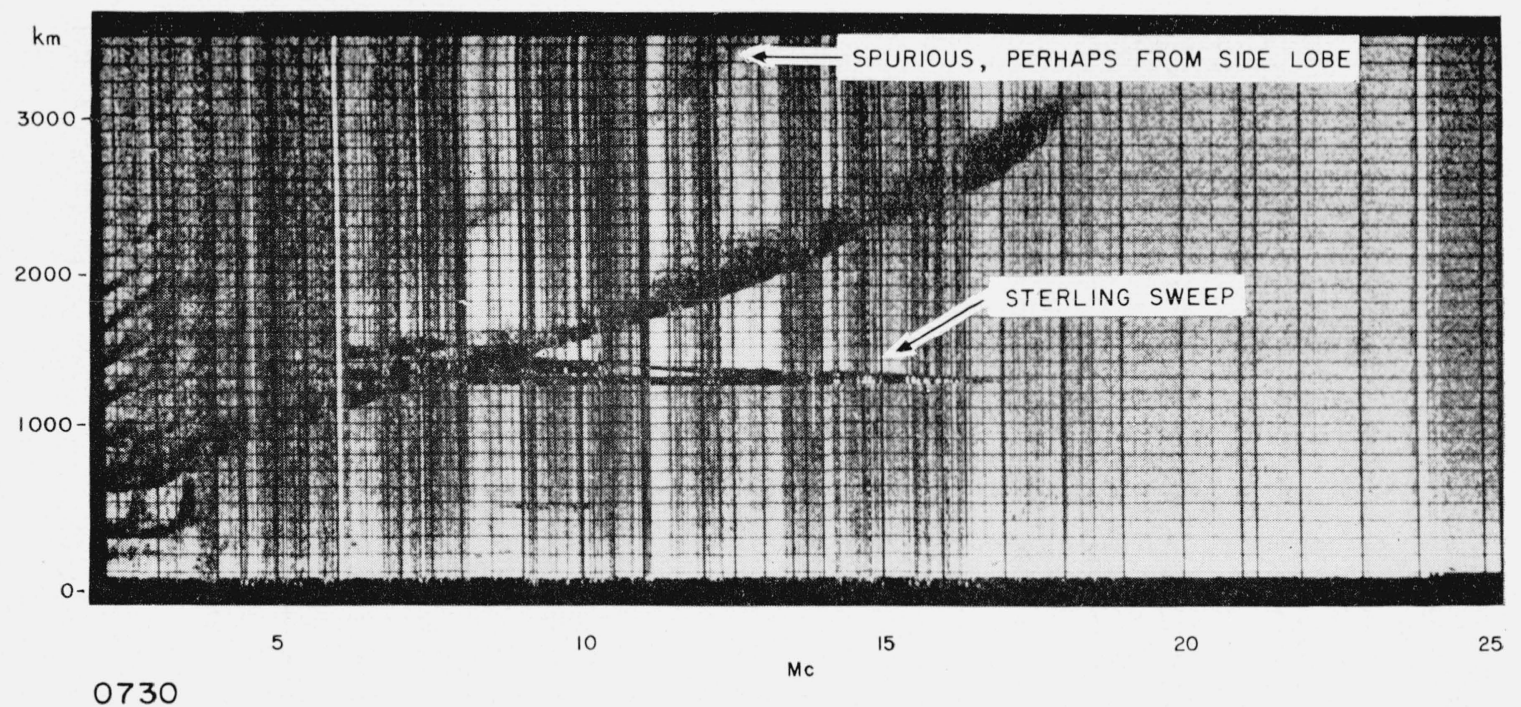

(a)

FEBRUARY 18,1955

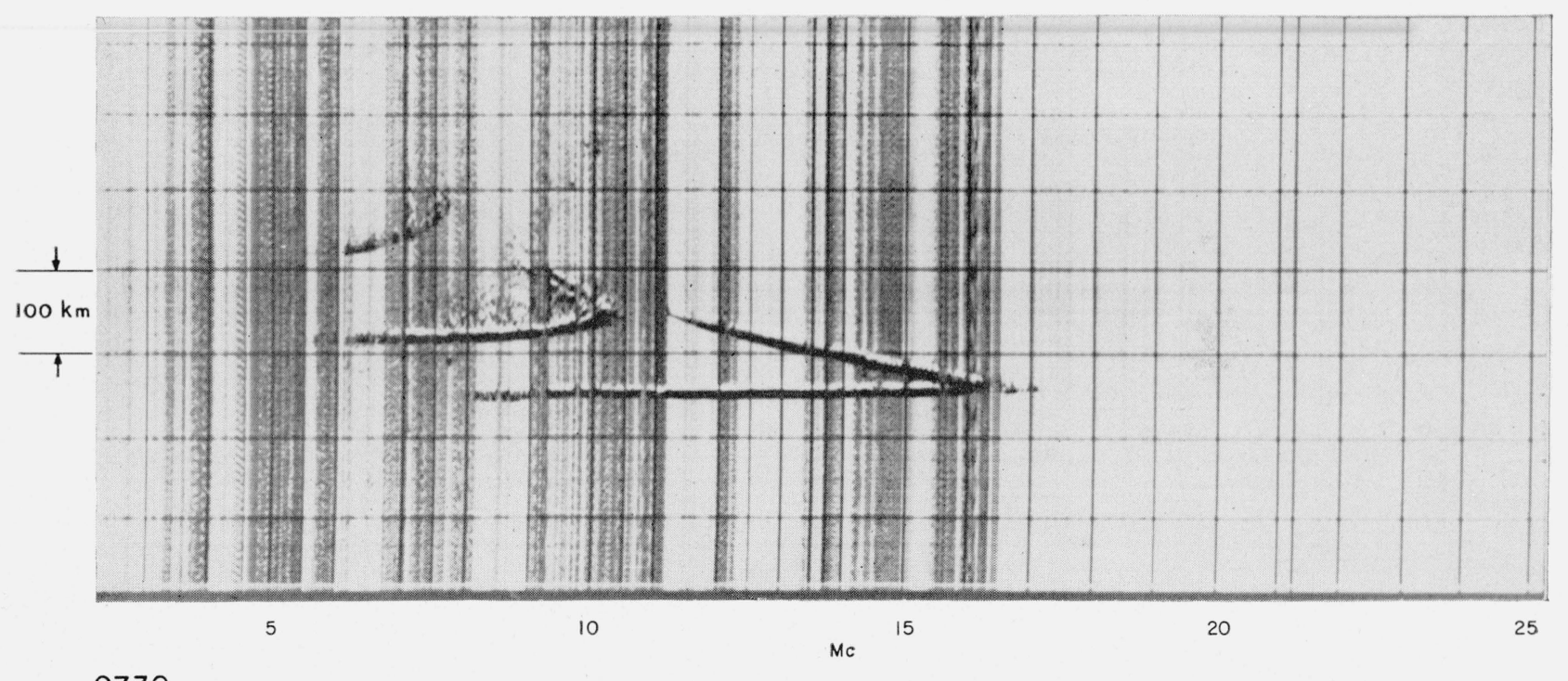

0730

(b)

Figure 12. (a) Backscatter record.

(b) Boulder to Sterling $P^{\prime} f$ sweep.

the critical frequencies are and where to place a transmission curve in order to derive the obliqueincidence MUF. A transmission curve is shown which, when placed on the vertical-incidence ionogram, gives a $1,150-\mathrm{km}$ MUF of $4.1 \mathrm{Mc}$. It appears that the transmission curve almost touches the inside edge of the spread echo rather than the high-frequency edge. It is of interest to note that there is no feature on the vertical-incidence ionogram giving a frequency of $5.1 \mathrm{Mc}$, which is the maximum fre- quency reflected from the $F 2$ layer at oblique incidence. Another feature of interest in figure 13a is the presence of sporadic- $E$ echoes on the obliqueincidence ionogram with no sign of echoes from the same layer at vertical incidence. A calculation of the separation between the Es trace and the $F 2$ trace indicates that the lower trace is, in fact, the one-hop Es trace.

A similar pair of records for the longer path is shown in figure 14. The transmission curves for 
MAY 29, 1952

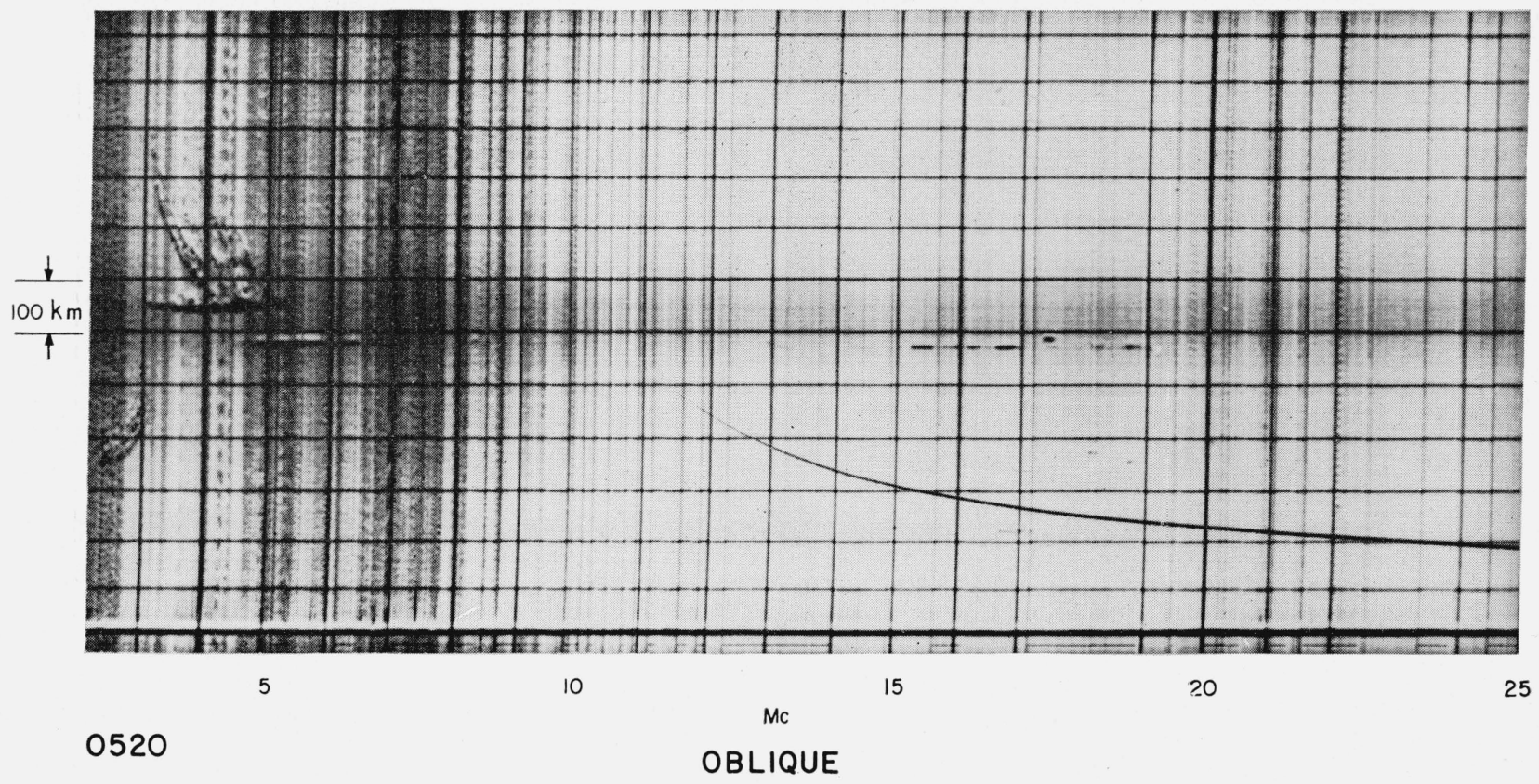

(a)

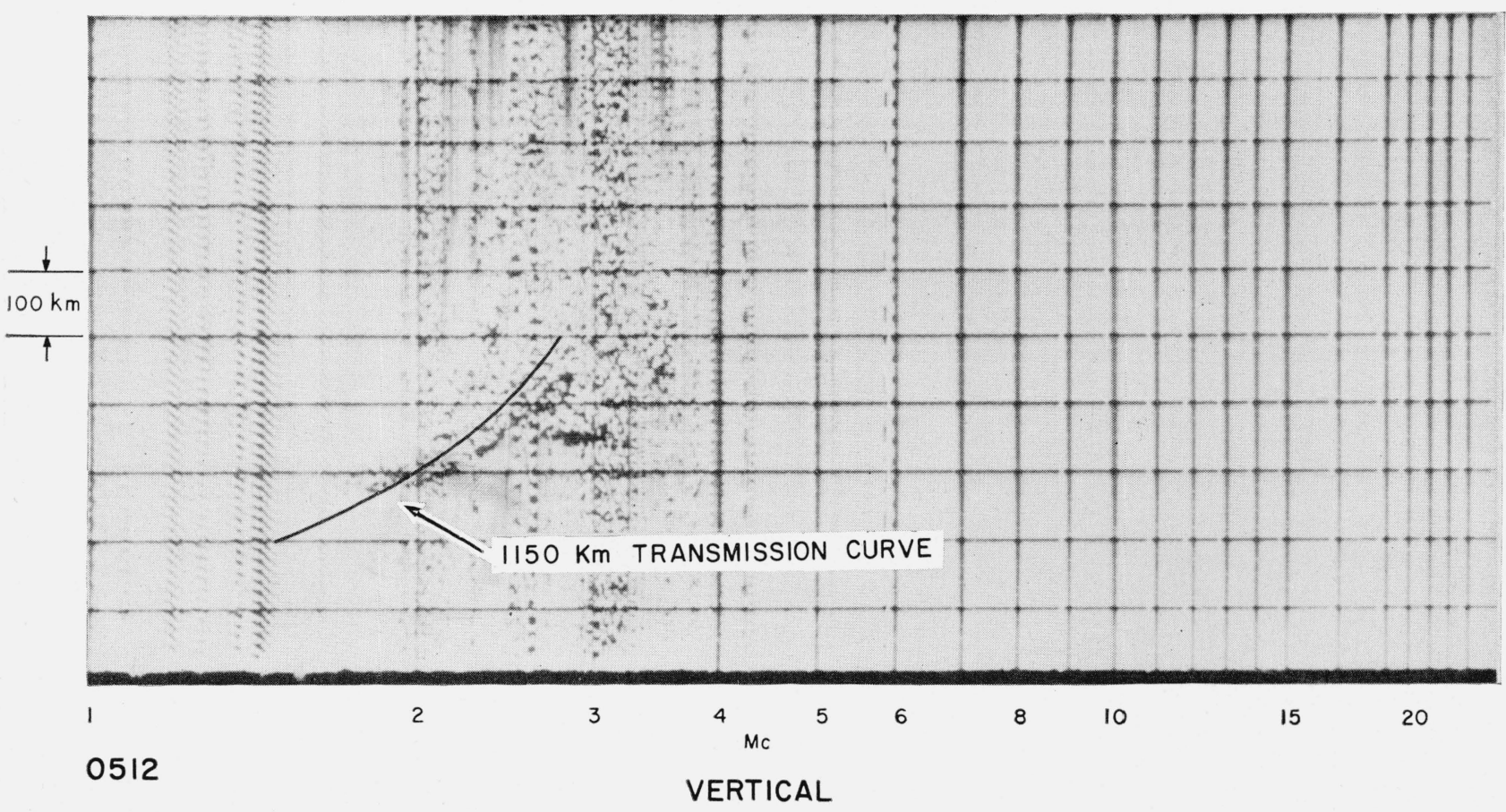

(b)

Figure 13. Scaling of the MUF in the presence of spread echo, Sterling to St. Louis. Note the proximity of the 4.1-Mc transmission curve to the inside edge of the spread echo. 
JANUARY 17,1955
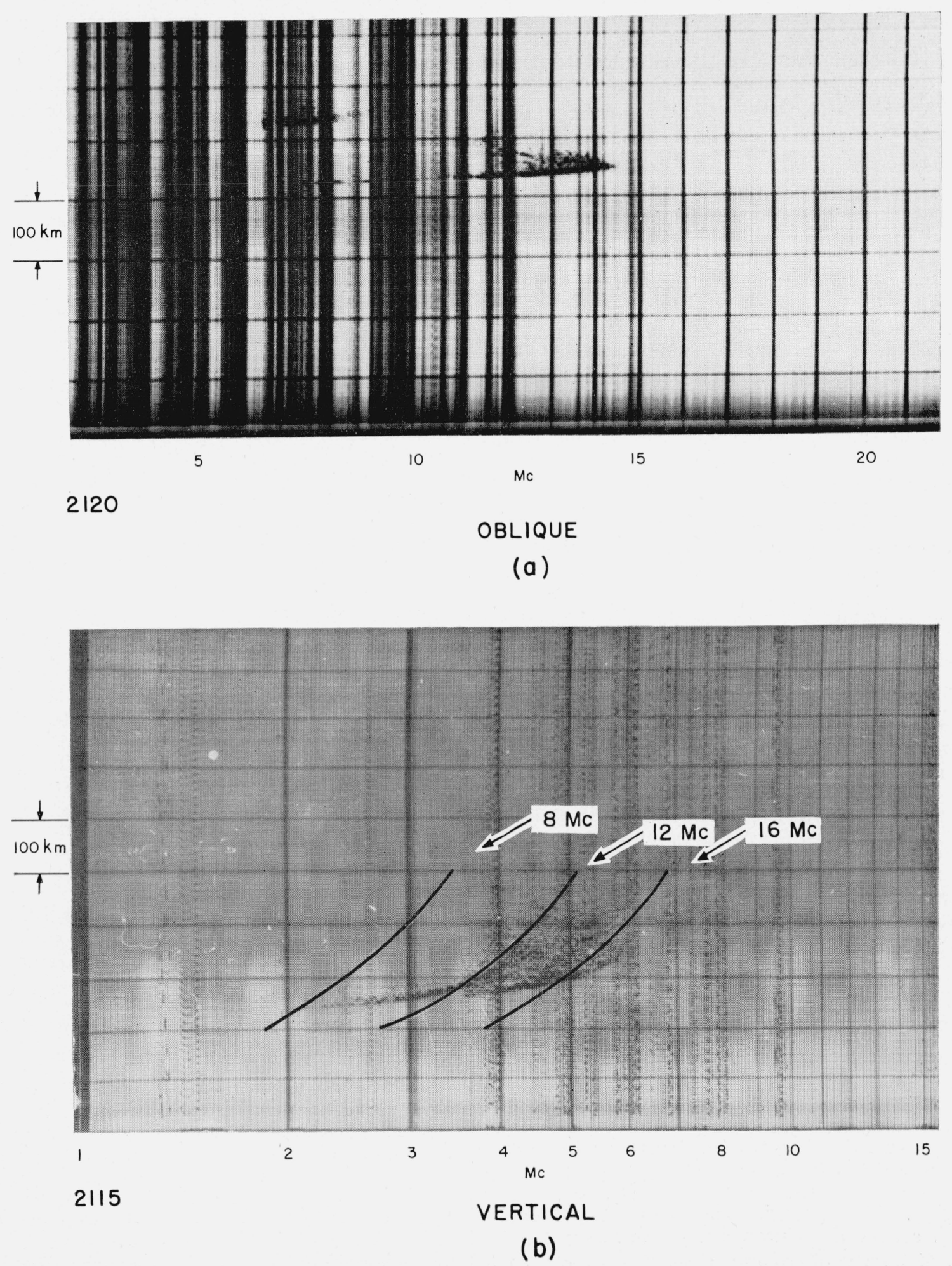

Figure 14. Scaling of the MUF in the presence of spread echo, Sterling to Boulder. 
8,12 , and $16 \mathrm{Mc}$ have been drawn across the midpoint record. The difficulties of scaling both of the records are apparent: it is not possible to match corresponding features. It should be made clear, however, that the discrepancy between the observed F2 MUF of about $14 \mathrm{Mc}$ and the calculated MUF of $12 \mathrm{Mc}$ (inside edge of spread) is no larger than occasionally arises when spread echo is not present.

\section{e. MUF Extensions}

It was pointed out that on the longer transmission distances echoes have been observed on frequencies higher than that at which the high-angle and lowangle rays join (the so-called classical MUF). Such extensions have been observed on both the 1,150-km and the 2,370-km paths in the United States.

In figure 15 are sample records taken over the $1,150-\mathrm{km}$ path showing two occurrences of MUF extensions, one on the $F 1$-layer trace by day and the other on the F2-layer trace in the early morning. In the sweep of 1146, April 3, 1952, we see that the junction of the high-angle and the low-angle rays cannot easily be determined but probably occurs at about 9.5 Mc whereas the maximum observed frequency for the $F 1$ trace is near $10.3 \mathrm{Mc}$. In this case there is difficulty in distinguishing between the $F 1$ trace and the two-hop $E$ trace. Note, however, the absence of any such extension on the ordinaryand the extraordinary- $F 2$ traces.

Sweeps showing MUF extensions on the 2,370-km path are shown in figure 16 . It will be seen that the extension has been observed both by day and by night. Figure $16 \mathrm{a}$ is a fairly typical winter-day sweep. We see that the high-angle and low-angle rays merge near 21.1 Mc whereas the maximum observed frequency is about $23.4 \mathrm{Mc}$. Even if we extrapolate the lower edge of the high-angle ray trace (about 22.0 Mc) there is still an appreciable extension of about 1.4 Mc. Figure $16 \mathrm{~b}$ is an early morning record which shows an MUF extension about 0.6 and 1.0 Mc depending on the procedure used in determining the MUF (see sec. 3a). It should be emphasized at this point that this phenomenon is not always observed. Figure 17 shows a relatively common sweep taken on the Boulder to Sterling circuit which shows no extension whatsoever.

The extension is greatest during winter days and is only occasionally observed during the middle of the winter nights. The diurnal variation of the median extension of the $F 2$ trace for the winter of 1954 to 1955 is shown in figure 18 . The extension is practically nonexistent between the hours of 2030 and 0230 , and there is a marked dip in the curve near noon. It may be relevant to note that the dip, which occurs between about 0800 and 1600 , is coincident with the solar enhancement of the $D$ and $E$ layer ionization. The extension may thus be influenced by the electron distribution below the $F 2$ layer.

The histogram in figure 10 for the longer path gives a median winter-day difference between maximum observed frequency and calculated MUF of about 7 percent. It has been pointed out above that whereas this median difference was higher than that indicated in figure 7 for the shorter path, figure 7 (for the Sterling to St. Louis path) is a plot of percentage difference between classical and calculated MUF's. Figure 18 is an indication of the reason for the major difference between figure 7 and figure 10 . The 4 percent to 5 percent median MUF extension from figure 18 may be applied as an approximate correction to the 7 percent difference seen in figure 10 . The corrected difference of about 3 percent agrees well with the short-path result given by Wieder. For the paths used in the Bureau experiments, it appears then that the error, i.e., the difference between the calculated MUF and the classical MUF, is independent of path length.

\subsection{Other Phenomena}

\section{a. High-Angle Ray}

For many years in radio communications forecasting the high-angle ray was neglected in comparison with the low-angle ray on the basis that, except near the maximum frequency, deviative $a b$ sorption and purely geometrical defocusing would be excessive. This argument is supported by sweeps such as that shown in figure 19 in which only the low-angle ray of the one-hop echo is observed. On the other hand, there are times when the high-angle ray may be important relative to the low-angle ray. Such conditions are especially prevalent during summer days as seen in figures $20 \mathrm{a}$ and $20 \mathrm{~b}$. In figure $20 \mathrm{a}$ we see that for $1,150 \mathrm{~km}$ the low-angle ray is completely absent for the one-hop mode, whereas the high-angle ray appears to be relatively strong and decreasing in delay time up to about $9.3 \mathrm{Mc}$; above that frequency the signal is cut off because of the increase in the angle of incidence. In figure $20 \mathrm{~b}$ we see that in the frequency range between 5 and $6 \mathrm{Mc}$ the high-angle ray (ordinary wave) appears to be much stronger than the low-angle ray, probably because of reflection of the low-angle signal by the intense sporadic- $E$ which can be seen at frequencies up to about $18 \mathrm{Mc}$.

This explanation for absence of the low-angle ray is illustrated in figure 21. The low-angle ray is incident on the sporadic- $E$ layer at a larger angle than the high-angle ray and may, therefore, be reflected by $E_{\text {s }}$ at substantially higher frequencies than the more steeply incident high-angle ray and will not, under these conditions, be received.

\section{b. Low-Frequency Cut-Off}

As the transmitted frequency is increased from its lowest value, we often notice that the first signals to appear at the receiver are those which have suffered two or more reflections at the ionosphere. Two cases of this phenomenon are shown in figures 22 and 23. The former is a case where over the $1,150-\mathrm{km}$ path sporadic- $E$ cuts off the one-hop signal almost completely, whereas the two-hop echo, which penetrates the $E$ region at a different locality and with a smaller angle of incidence, comes in strongly above 


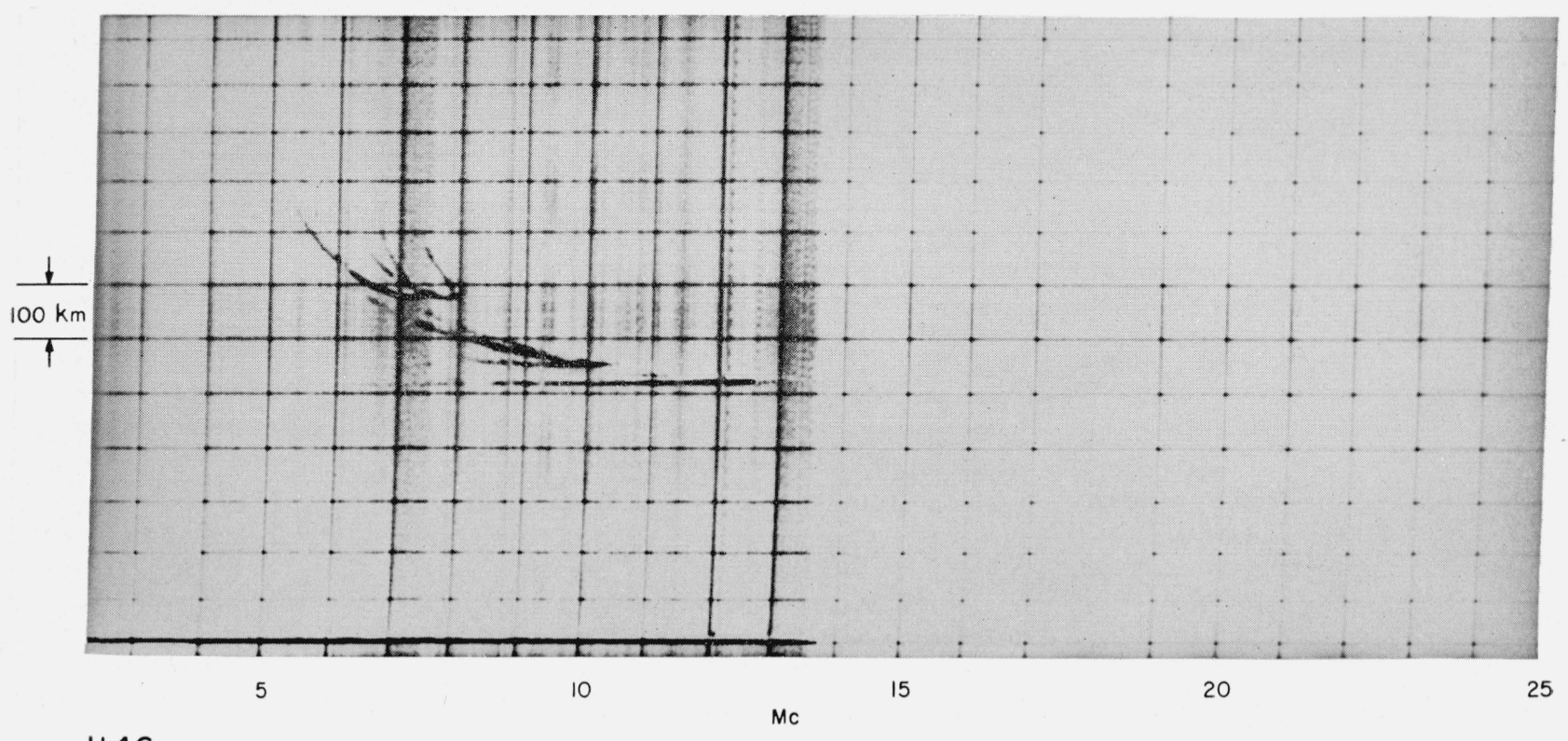

1146

(a)

SEPTEMBER 12, 1952

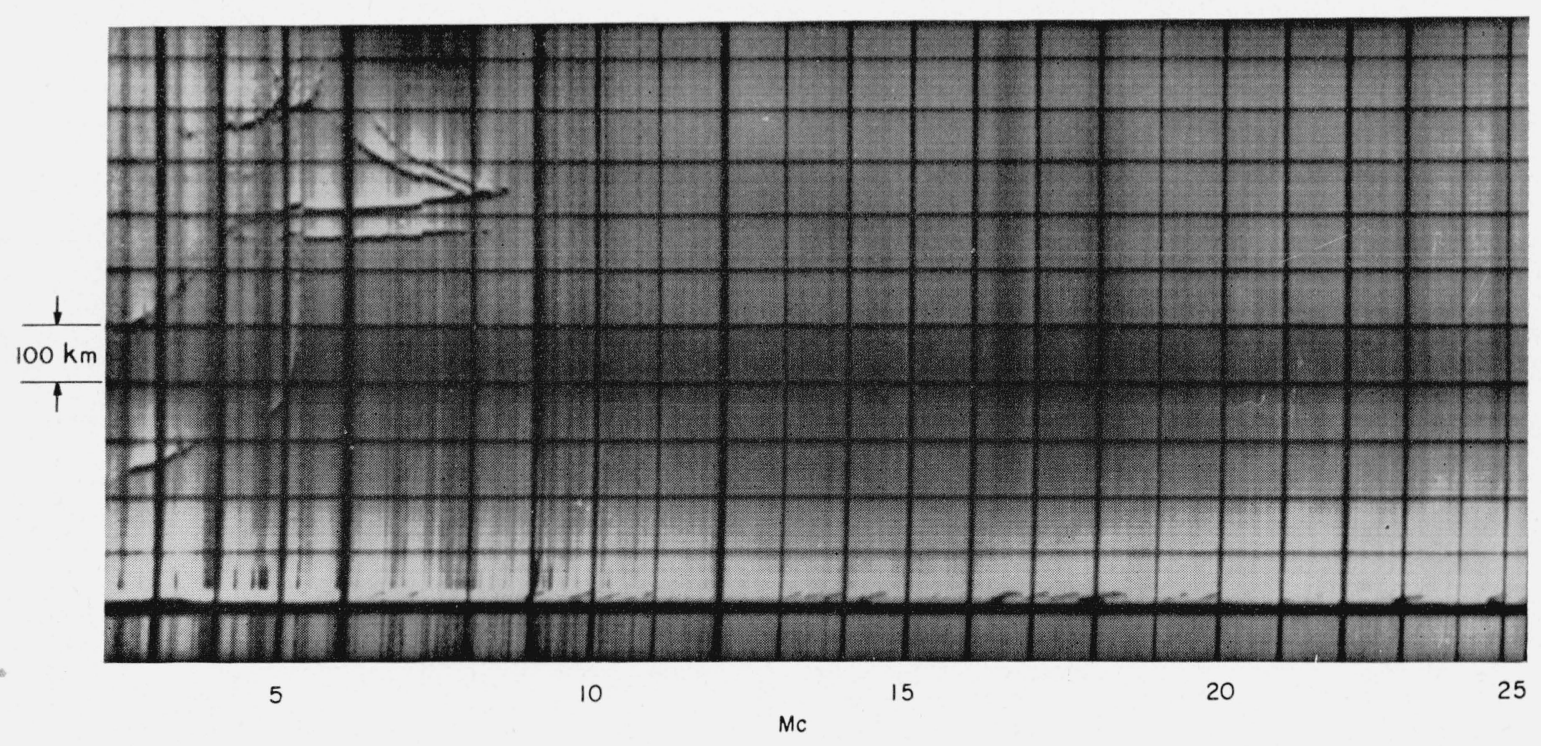

0652

(b)

FIGURE 15. Sterling to St. Louis sweeps showing the presence of MUF extensions. 
DECEMBER 7, 1954

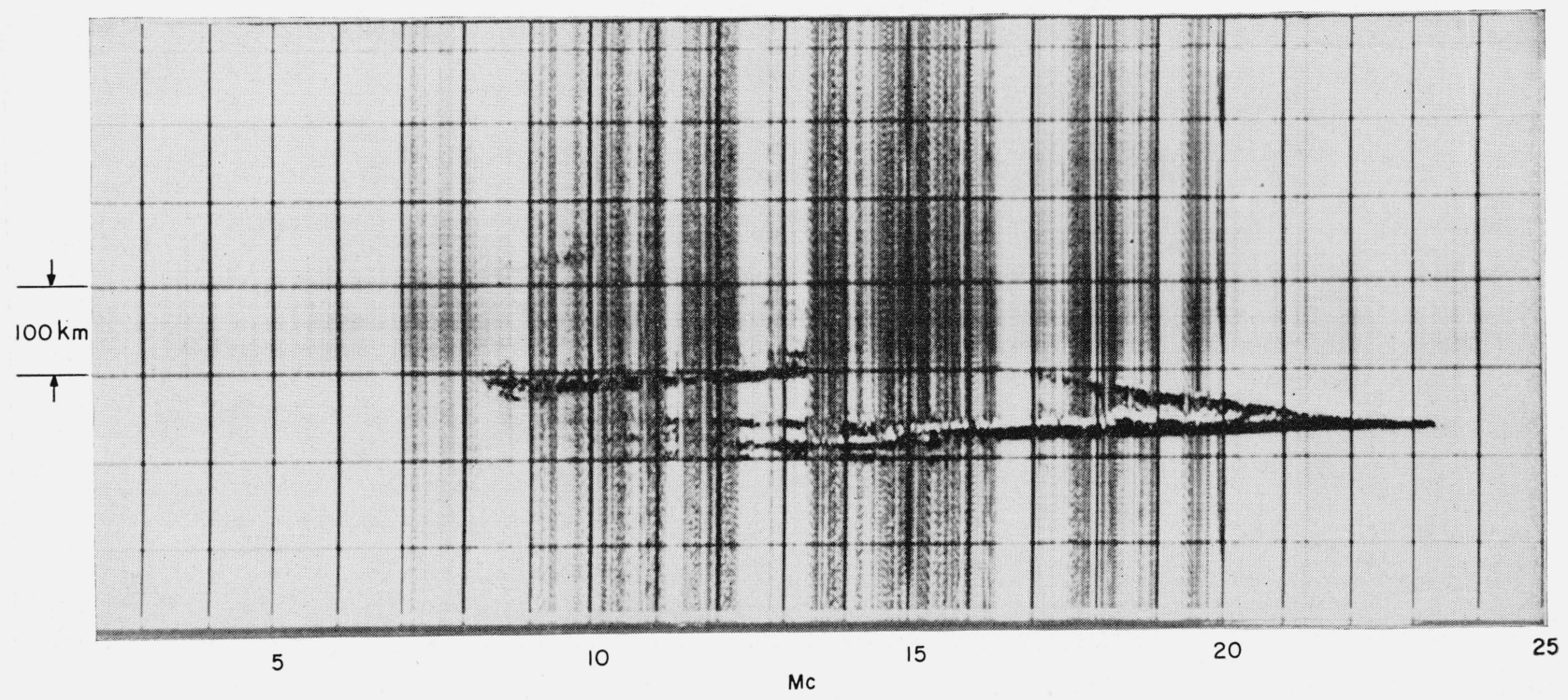

1123

(a)

OCTOBER 5,1954

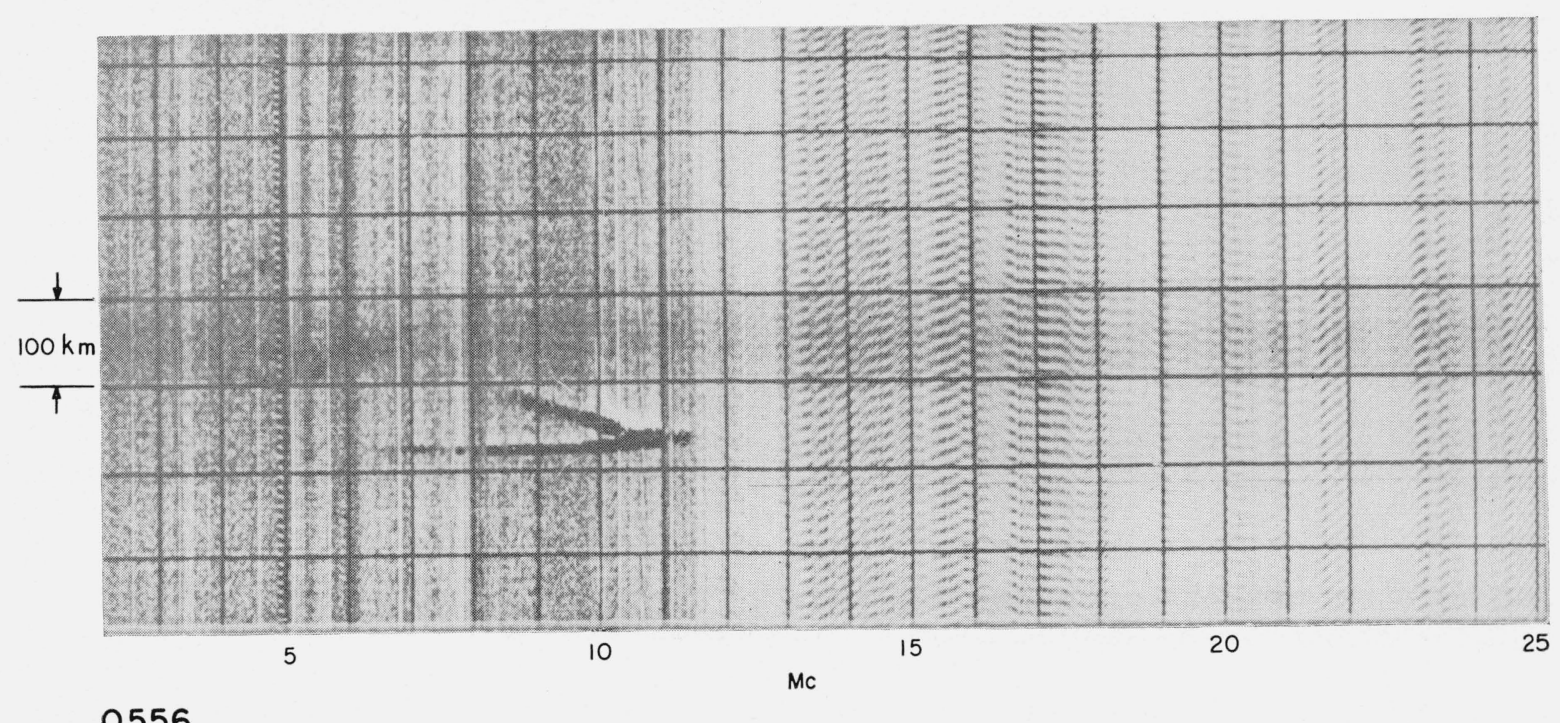

(b)

Figure 16. Sterling to Boulder sweeps with MUF extensions. 


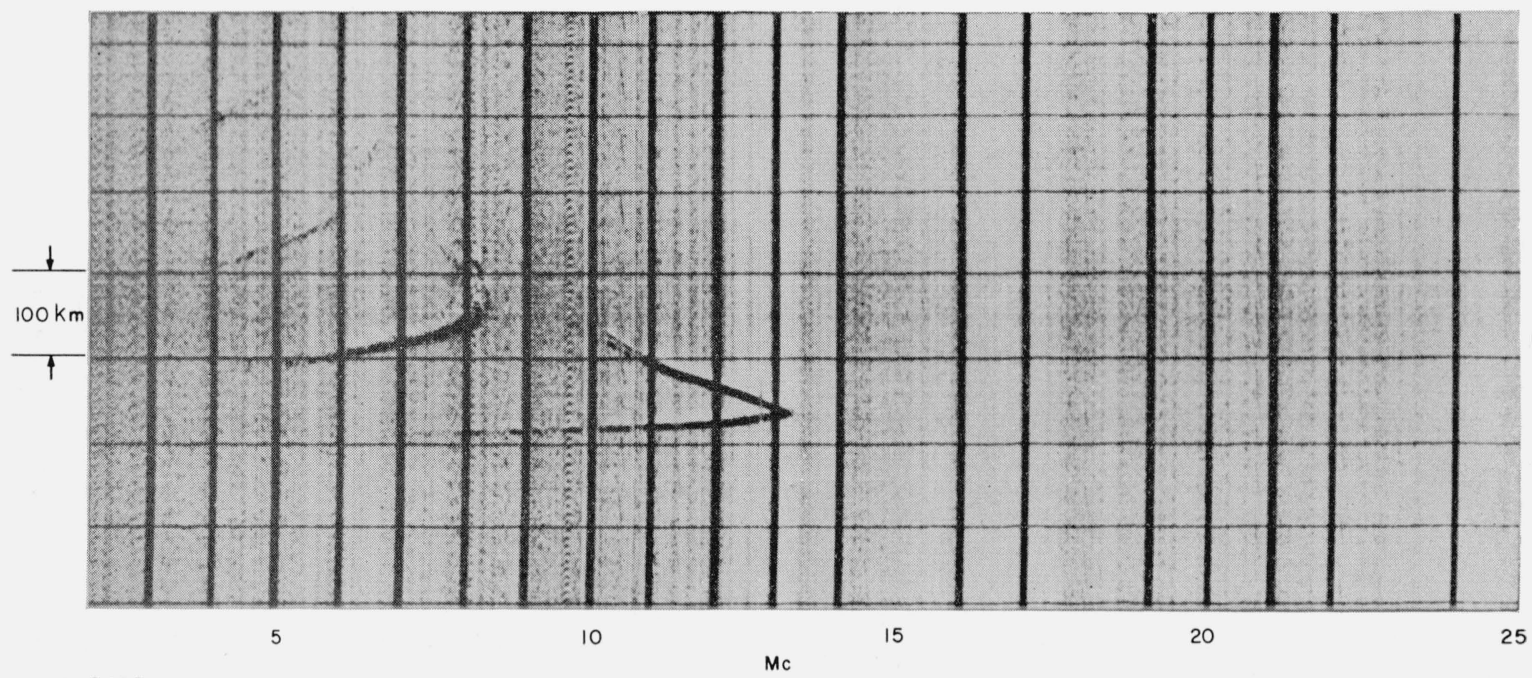

2112

Figure 17. Sterling to Boulder sweep without the MUF extension.

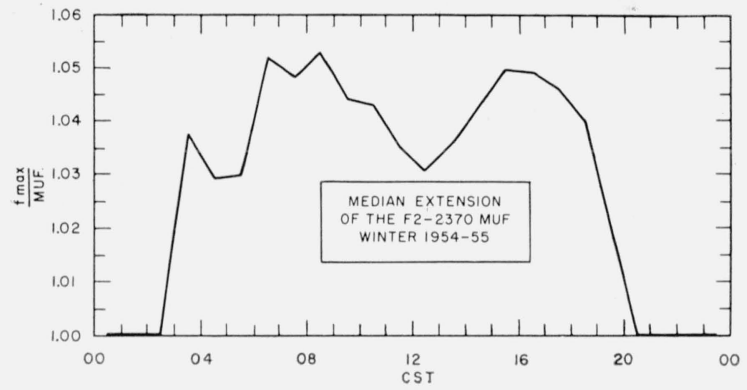

FIgure 18. The winter diurnal variation of the percentage MUF extension for the Sterling to Boulder path.

NOVEMBER 18,1952

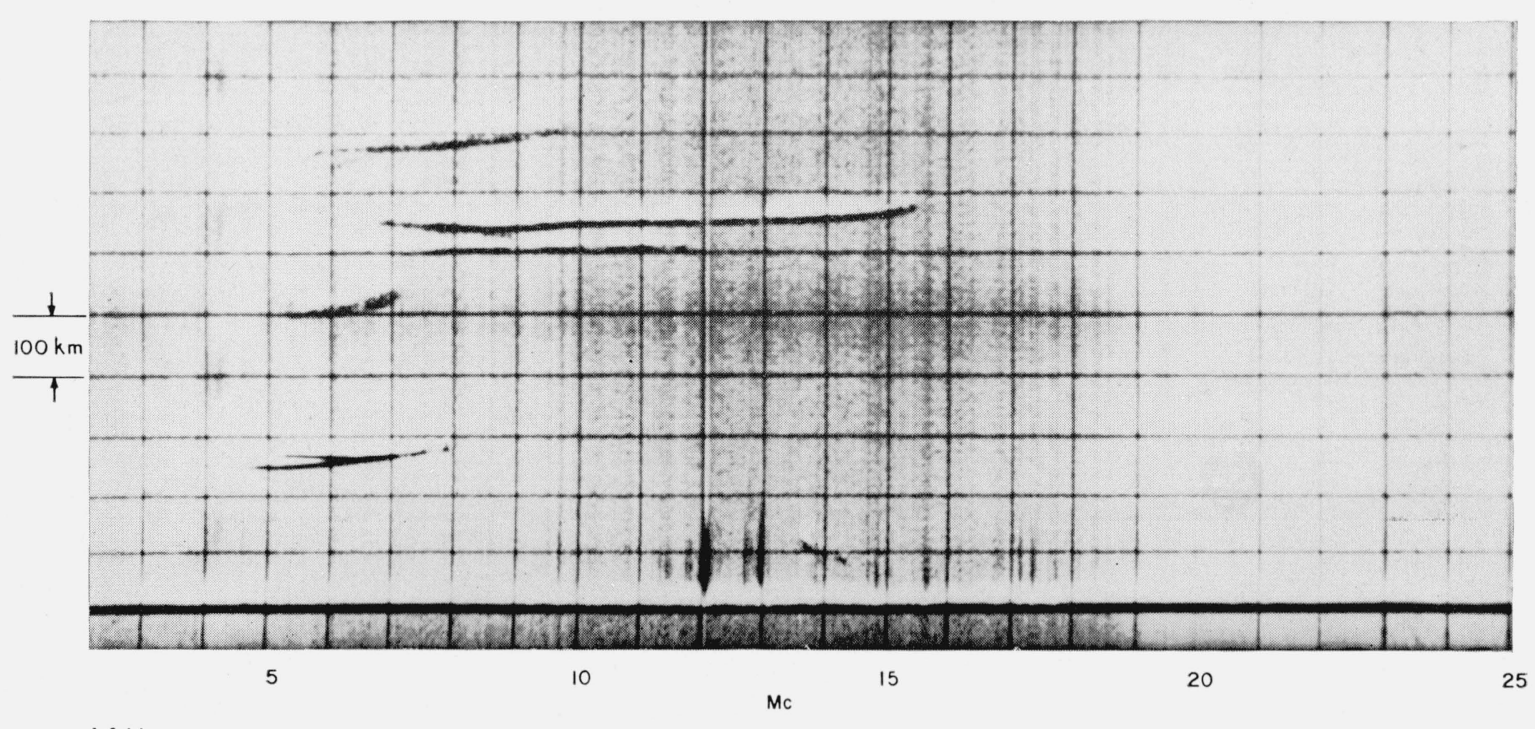

1411

Figure 19. Winter sweep showing the absence of the Pedersen ray signal over the Sterling to St. Louis path. 


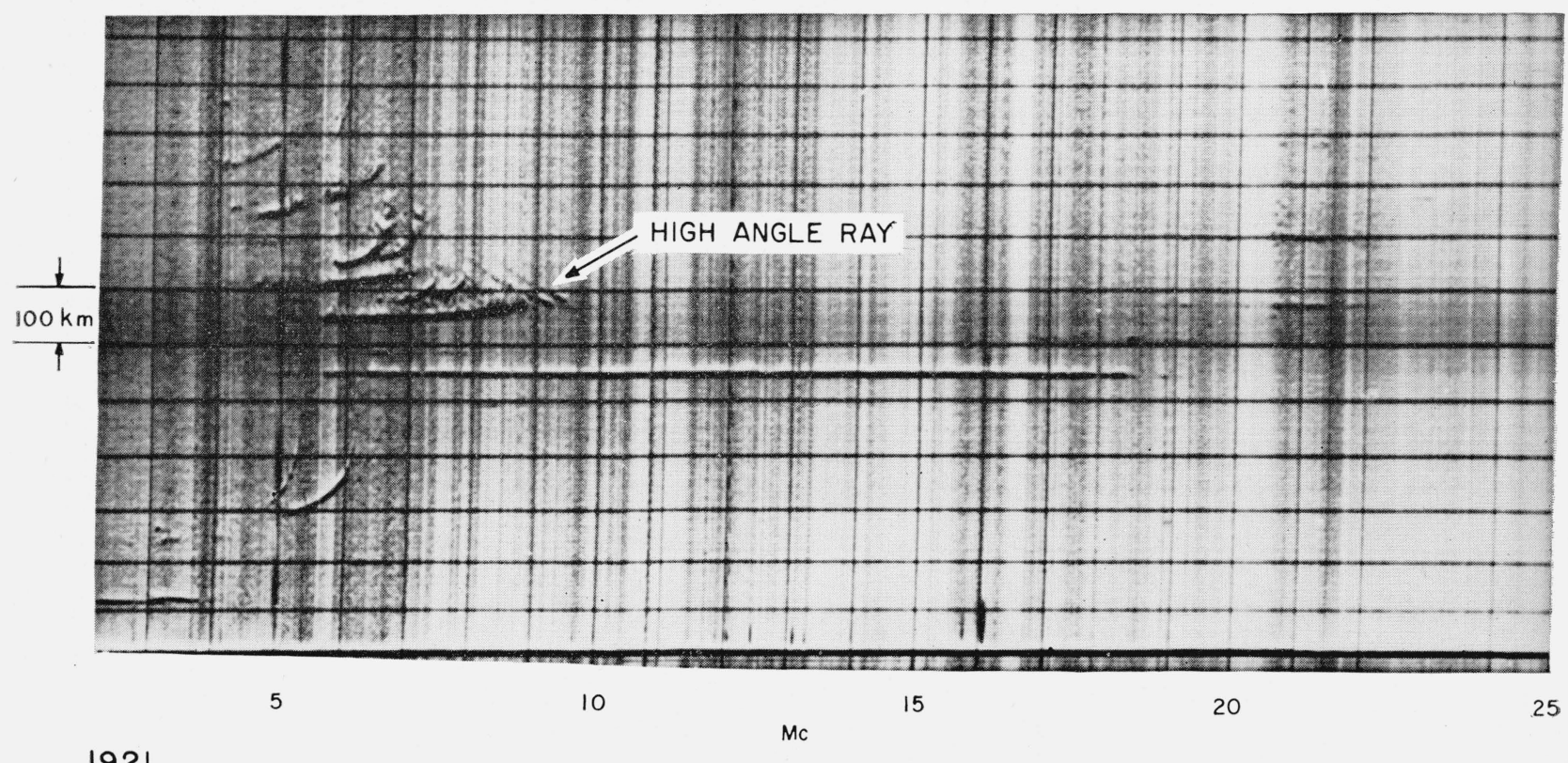

(a)

MAY 15, 1952

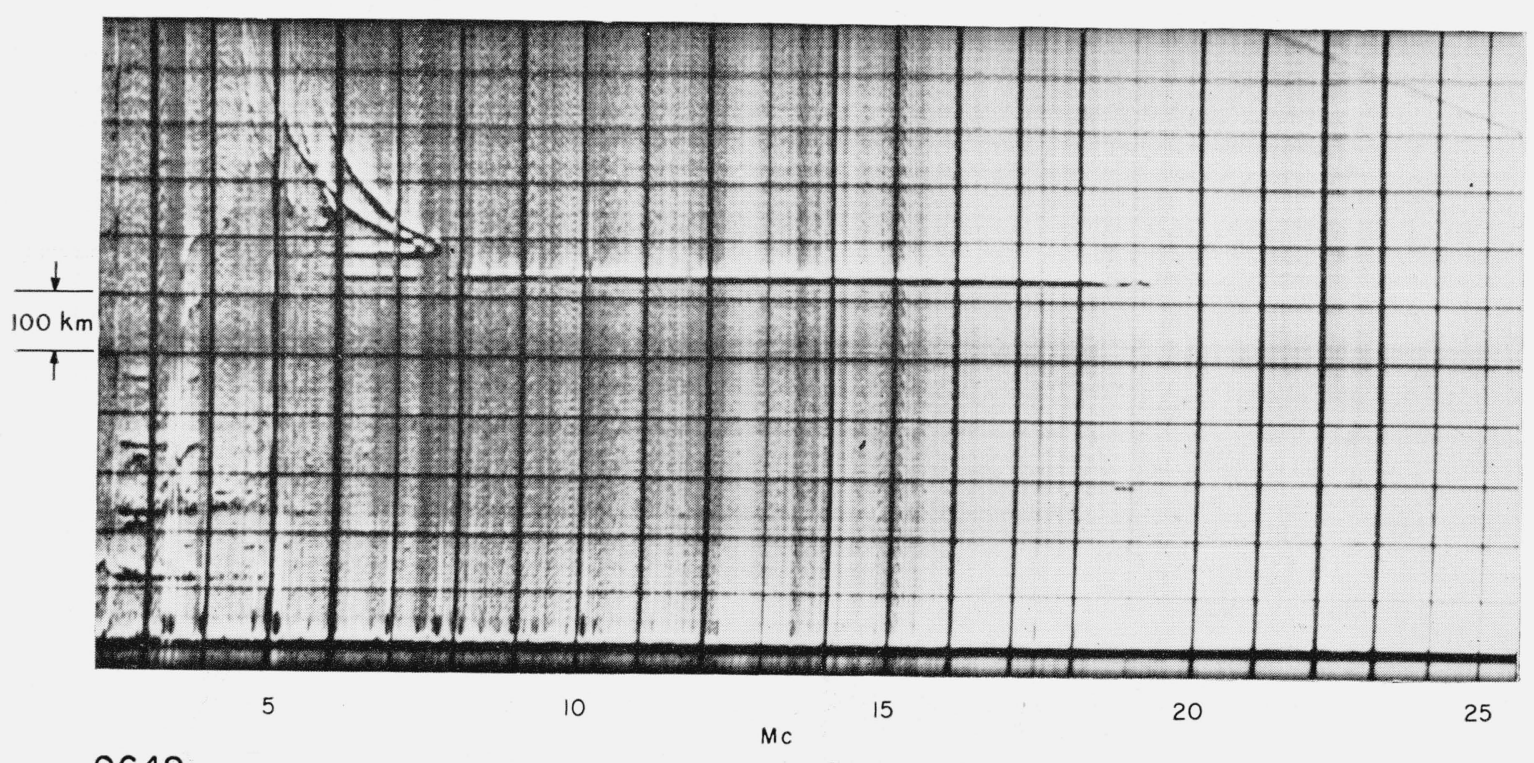

(b)

FIGURE 20. Sterling to St. Louis sweeps with examples of relatively strong Pedersen rays. 


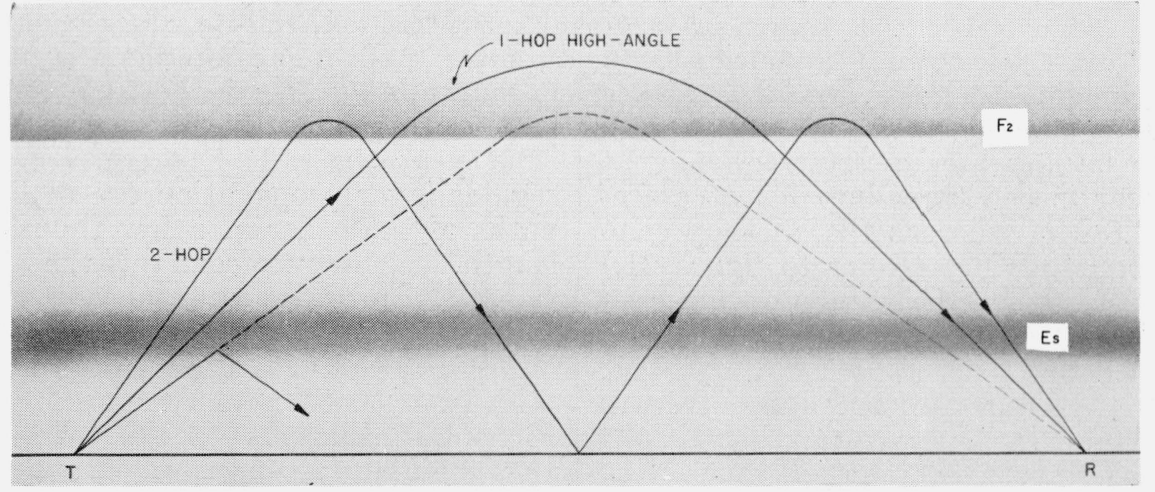

FIGURE 21. Illustration of the way in which the 1-hop low-angle ray may be cut off by the E layer while the Pedersen ray and the 2-hop mode can propagate between transmitter $(T)$ and receiver $(R)$.

MAY 15, 1952

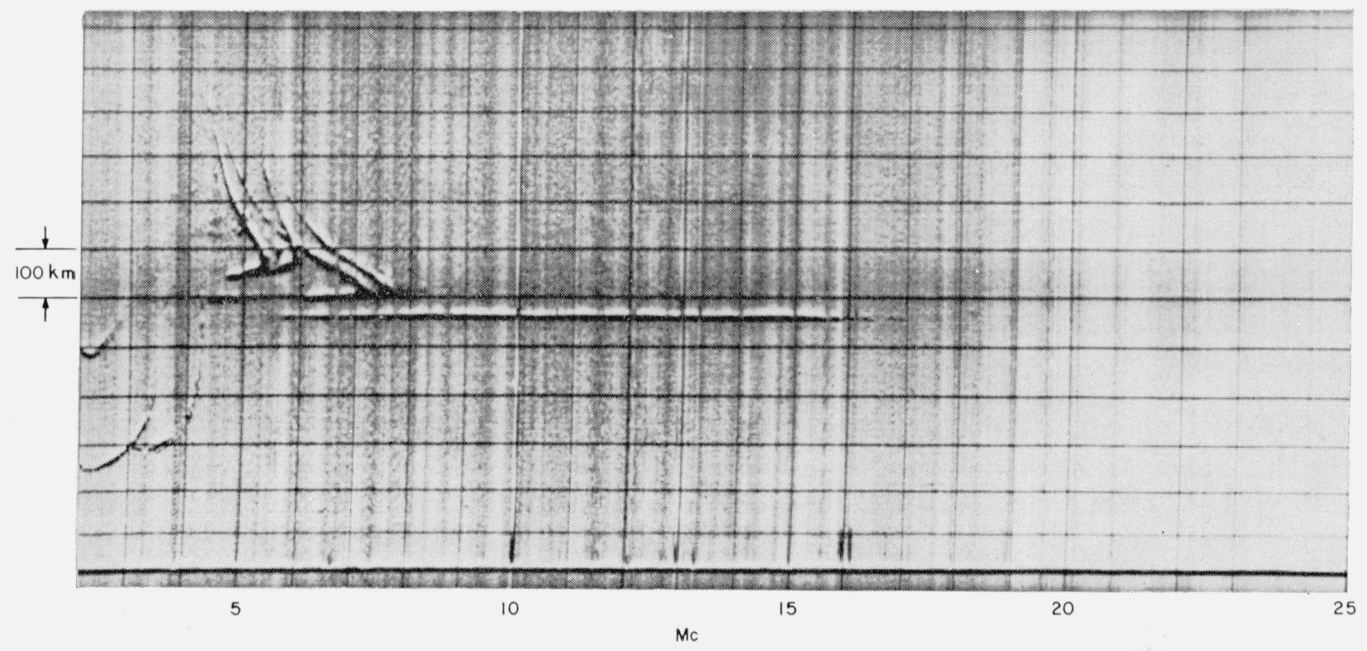

0637

Figure 22. Low-frequency cut-off on the Sterling to St. Louis path.

DECEMBER 20, 1954

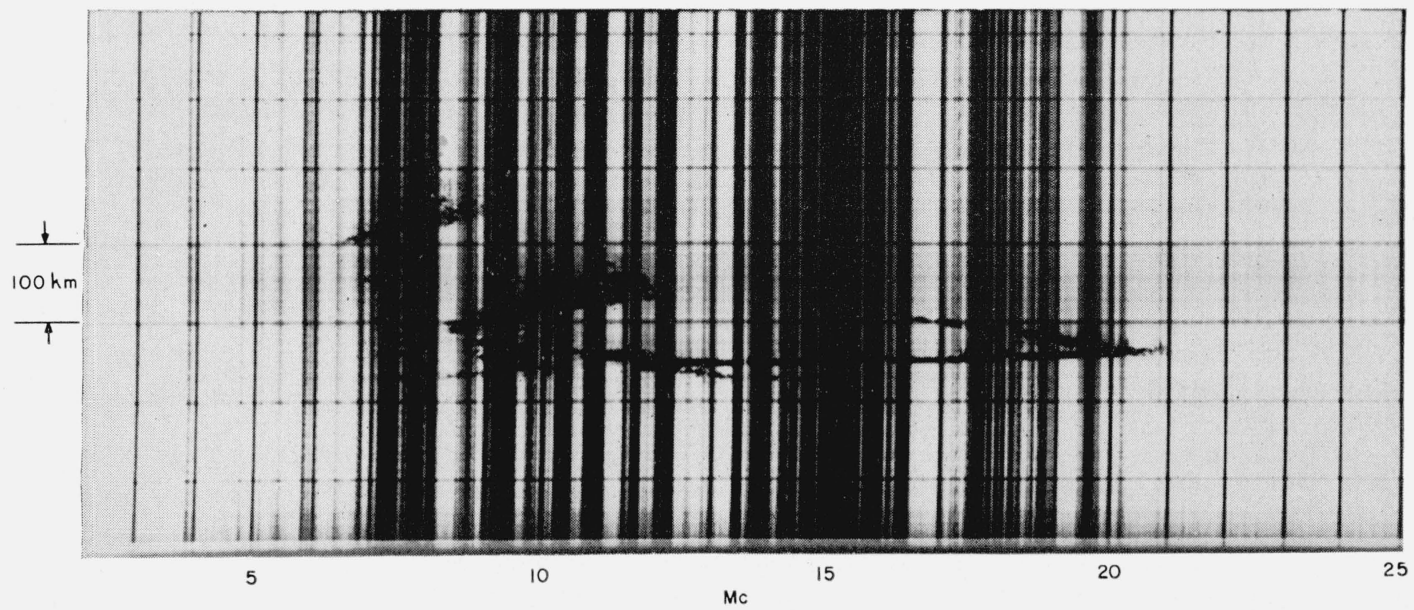

1024

Figure 23. Low-frequency cut-off on the Sterling to Boulder path. 
4.7 Mc. It will be seen that the high-angle rays of both modes have lower observed frequencies than the corresponding low-angle rays. Figure 23 shows the same phenomenon for the $2,370-\mathrm{km}$ path. We see that the trace having the lowest frequency at which echoes appear is the three-hop $F 2$ at a frequency of about $6.8 \mathrm{Mc}$, whereas the one-hop echo first appears at about $9 \mathrm{Mc}$. (Refer to figure 21.) Sulzer [15] attributed this phenomenon to antenna patterns but the lowest observed frequencies are in good agreement with the $E$-layer cut-off hypothesis, and we conclude that the effect of antenna pattern is of secondary importance.

\section{c. Separation of the Ordinary and Extraordinary Rays at the MUF}

As the distance of transmission is increased, the maximum frequencies of both the ordinary and the extraordinary waves are increased. However, the separation between the two MUF's decreases with increase of distance. This frequency separation depends upon the angle of incidence; the frequency; the strength of the magnetic field; and, to some extent, on the direction of propagation with respect to the magnetic field.

In figure 24a we see that the effect of refraction in the lower layers is to increase the frequency separation of the ordinary and extraordinary MUF's. This is a result of a decrease in the angle of incidence of the ray at the $F 2$ layer. The separation is about $0.6 \mathrm{Mc}$.

In figure $24 \mathrm{~b}$ we see the effect of angle of incidence by comparing the MUF separations for the one-hop and two-hop modes of propagation where the separations are about 0.3 and $0.6 \mathrm{Mc}$, respectively. Part of this change may be due to the lower maximum frequencies of the two-hop signals since even at vertical incidence the critical frequency separations between the ordinary and extraordinary rays increase as the critical frequencies decrease. This argument cannot be applied to the case in figure 24 a since the F2-layer MUF's in both figures 24a and 24b are practically equal (around $7.2 \mathrm{Mc}$ ). Note in the one-hop mode how for a given ground range the frequency separation increases with increase of group path.

A statistical analysis has been made to find the mean separation of the MUF's for east-west propagation in the United States. The separations refer to the one-hop mode for the $2,370-\mathrm{km}$ path, the onehop and two-hop modes for the $1,150-\mathrm{km}$ path and the vertical-incidence data. The results are plotted in figure 25, where it is seen that, on the average, the separation falls off steadily with distance from about $0.8 \mathrm{Mc}$ at vertical incidence to about $0.2 \mathrm{Mc}$ at $2,500 \mathrm{~km}$.

\subsection{Discussion of the NBS Results}

The experiments described above have shown that over the distances of transmission concerned $(1,150$ and $2,370 \mathrm{~km}$ ) the $F 2$ layer is not always the layer which determines the MUF. Sporadic- $E$ is of particular importance on the shorter path and usually determines the MUF during the sunlit periods of the summer months. Sporadic- $E$ is not only important in determining the MUF but it may also decide the lowest observed frequency because of cut-off. Thus it is clear that MUF predictions based solely on regular layer propagation are of limited value over paths of the order of a few thousand kilometers in length. It follows from this that tests of the accuracy of regular layer prediction methods based on times of fade-in and fade-out of $C W$ signals may be highly misleading. Again, during the summer daylight periods, the $F 1$ layer is important in deciding the MUF especially on the $2,370-\mathrm{km}$ path because the one-hop $E$ reflection is generally nonexistent. The $F 1$ layer is relatively more important during periods of low sunspot activity when daytime foF2 sinks to relatively low values and also during magnetically disturbed periods.

The F2 layer is, however, the dominating layer by night at all seasons and throughout the winter day except for short periods when sporadic- $E$ may be present.

Perhaps the most important aspect of the obliqueincidence experiments is the relationship between vertical-incidence data and oblique-incidence data. The first feature of interest is the close correlation between the temporal changes (both long and short term) between the observed MUF's and the calculated MUF's. The calculated MUF's are, on the average, some 3 percent lower than the observed values. This percentage difference is fairly consistent especially during the winter months. The source of this systematic error must lie in the assumptions in the theory underlying the Smith transmission curves. The essential assumptions made in the calculation of these curves are: 1 . The geometric optical approximation is valid. 2. The influence of the earth's magnetic field can be neglected. 3. The effect of ionosphere curvature can be accounted for by a correction factor $k$ which varies with distance of transmission only. 4. The ionosphere is spherically stratified. 5. Tropospheric refraction is negligible. The first assumption, i.e., the applicability of geometric optics, probably needs some modification near the MUF. This is especially so in the case of pulse propagation where the signal is composed of components spread over a fairly wide frequency range. For instance, when the carrier frequency is at the ray MUF, half the frequency components have frequencies above the MUF. Some modification of the variation of amplitude with frequency near the MUF will result from the application of wave theory but the effect on the value of the MUF is probably slight.

The influence of the earth's magnetic field on the ray path is usually small over the frequency range covered by the oblique-incidence work described above, except during the winter nights when the MUF falls to around 3 to $4 \mathrm{Mc}$. At these frequencies the frequency separation between the ordinary and extraordinary MUF's shows a noticeable increase over the separation at the higher frequencies. The important thing about the effect of the magnetic 


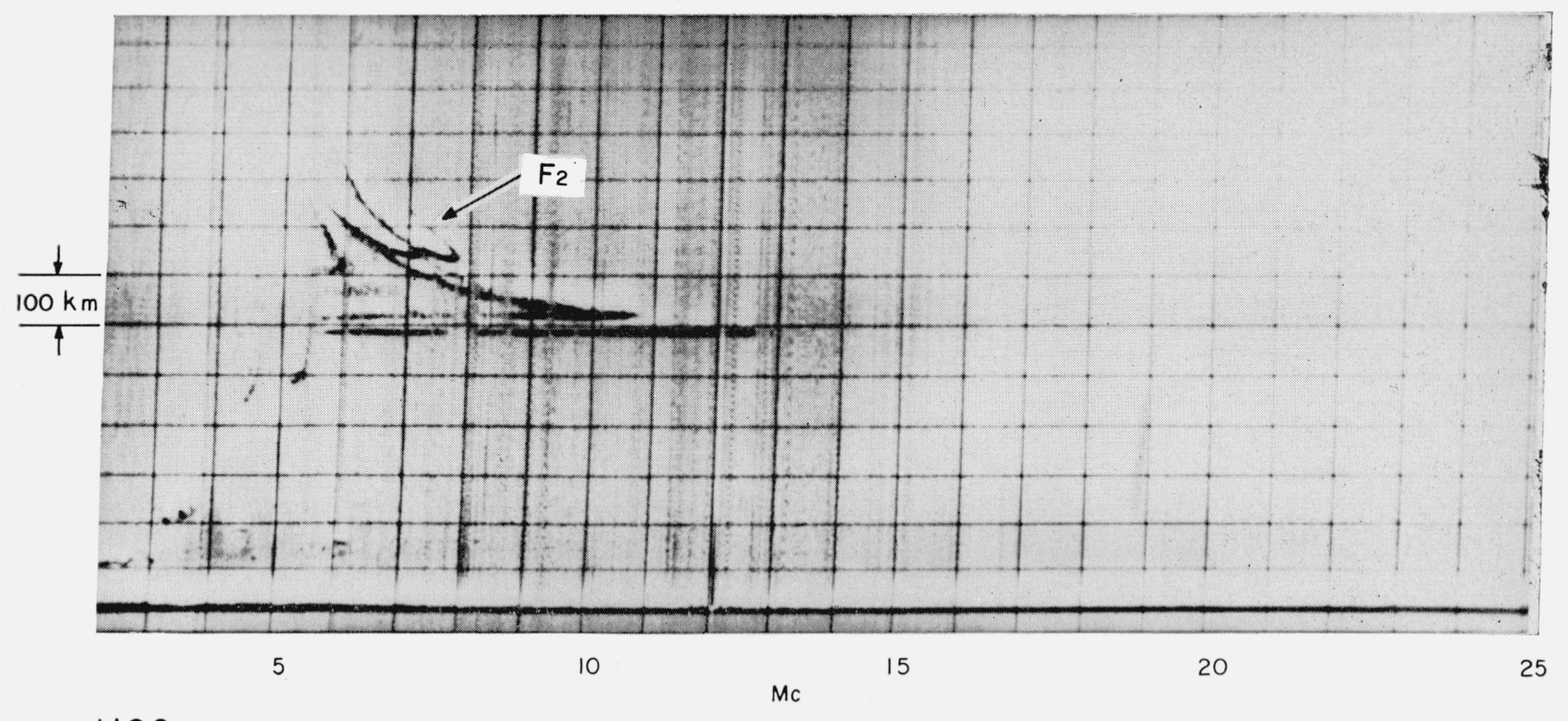

1120

(a)

MAY 15, 1952

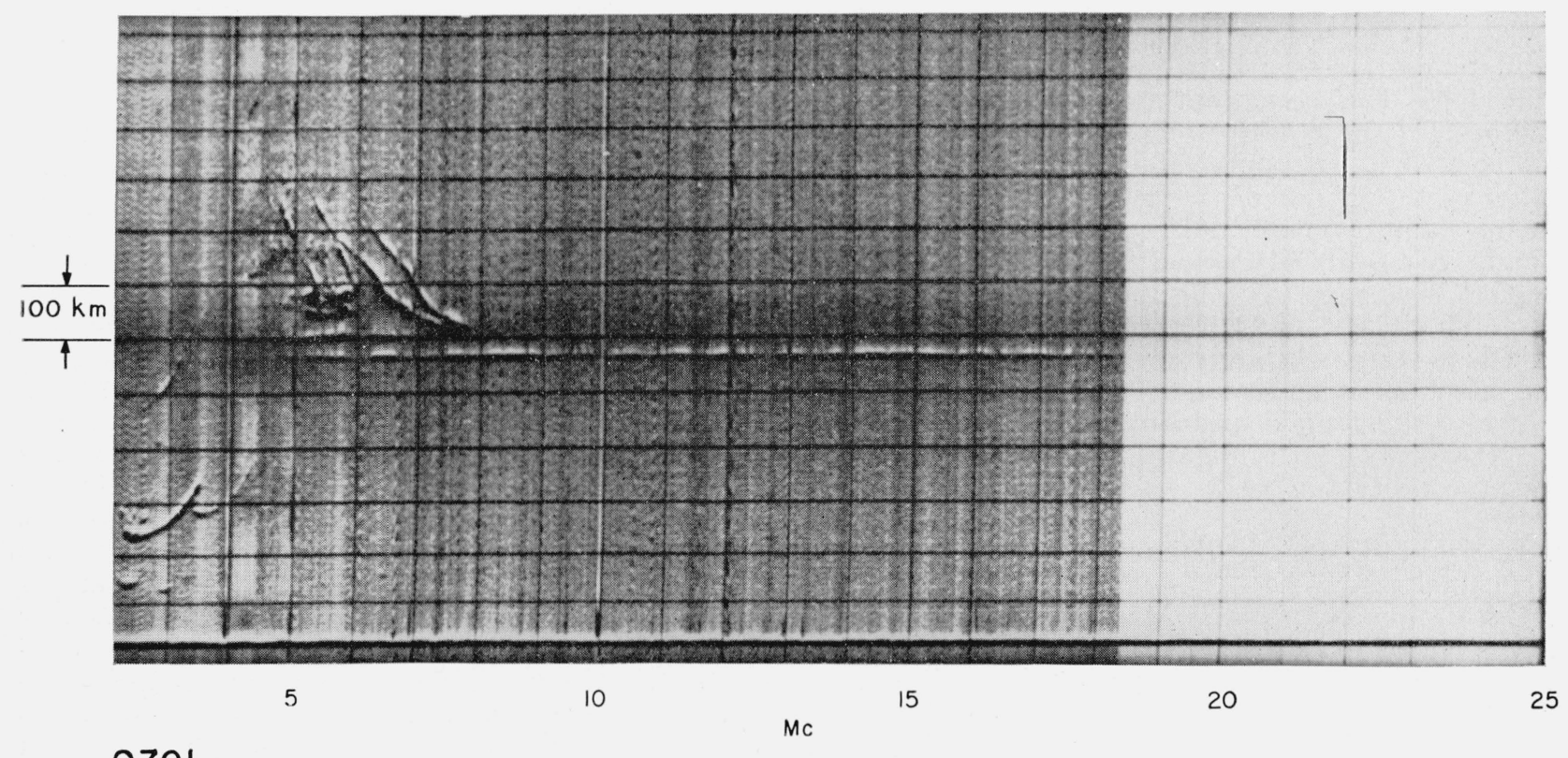

(b)

FIGURE 24. Sweeps showing the dependence of the frequency difference between the ordinary and extraordinary MUF's on group retardation and angle of incidence for the Sterling to St. Louis path.

Note how the frequency separation increases as the virtual height increases. 


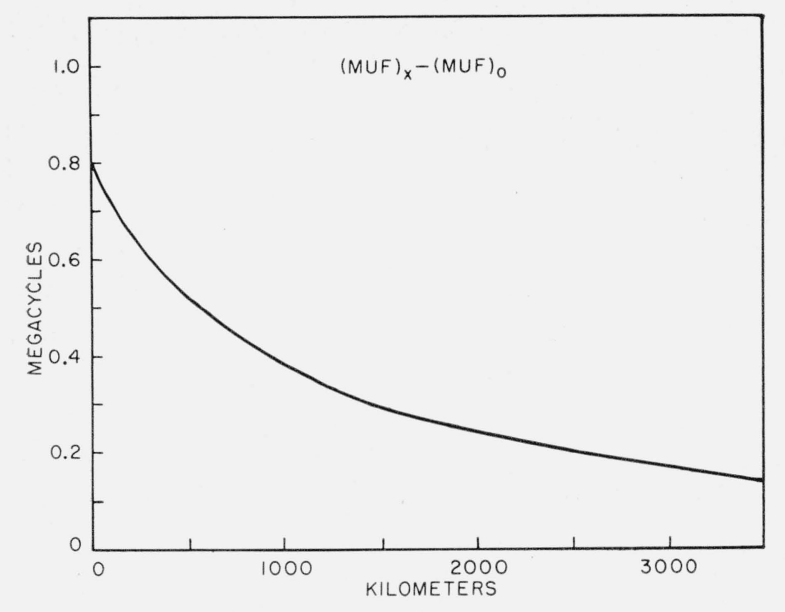

Figure 25. Variation with distance of the observed difference between the ordinary and extraordinary ray $M U F^{\prime} s$ for eastwest propagation in the United States.

field, however, is that it always tends to increase the ground range of the ordinary wave as compared with the case with no magnetic field. In other words, the effect of the field is always to reduce the MUF which is contrary to the observed phenomenon. The only exception to this is the case of east-west propagation along the magnetic equator where the MUF is unaffected by the magnetic field.

Wieder [14] has suggested magnetoionic deviation coupled with short-term variations in ion densities (and their geographical and temporal distributions) as a possible cause of the dispersion of the differences existing between the calculated and measured MUF's. Such an explanation holds, of course, only if the point of reflection for the oblique ray is located at an appreciable distance from that for the vertically incident ray (at an equivalent frequency) and if, in addition, appreciable horizontal gradients in ion density sometimes exist in the distance that separates the reflection points.

It is diffic ult, because of the number of parameters involved, to state with any generality the magnitude of the effects of magnetoionic deviation. However, insight into the general problem is given by application of Booker's [24] semigraphical method for the following conditions:

a. Horizontally stratified parabolic layer of 100$\mathrm{km}$ semithickness;

b. Gyrofrequency, $f_{H}=1.5 \mathrm{Mc}$;

c. Magnetic $\operatorname{dip}=70^{\circ}$;

$d$. Vertical plane containing direction of phase propagation perpendicular to vertical plane containing magnetic field direction (east-west propagation);

$e$. Angle of incidence for oblique ray, $\theta=60^{\circ}$;

$f$. Frequency $(f)$, propagated obliquely $=15 \mathrm{Mc}$ $\left(f_{H} / f=0.1\right) ;$ and

$g$. Vertical incidence (equivalent) frequency, $f$ $\cos \theta=7.5 \mathrm{Mc}\left(f_{H} / f=0.2\right)$.
The results of the computation of the magnetoionic deviation for layers having ordinary wave critical frequencies $\left(f_{0}\right)$ of 7.5 and $10 \mathrm{Mc}$ are given in table 1 .

TABLE 1

\begin{tabular}{|c|c|c|c|c|c|c|c|c|}
\hline & \multicolumn{4}{|c|}{ Ordinary ray } & \multicolumn{4}{|c|}{ Extraordinary ray } \\
\hline & \multicolumn{2}{|c|}{$\begin{array}{l}\text { Vertical inci- } \\
\text { dence }\end{array}$} & \multicolumn{2}{|c|}{$\begin{array}{l}\text { Oblique inci- } \\
\text { dence }\end{array}$} & \multicolumn{2}{|c|}{$\begin{array}{l}\text { Vertical inci- } \\
\text { dence }\end{array}$} & \multicolumn{2}{|c|}{$\begin{array}{l}\text { Oblique inci- } \\
\text { dence }\end{array}$} \\
\hline $\begin{array}{l}f_{0}=7.5 \\
f_{0}=10\end{array}$ & $\begin{array}{c}H \\
100 \\
33.9\end{array}$ & $\begin{array}{c}D \\
46 \\
4.3\end{array}$ & $\begin{array}{c}H \\
100 \\
33.9\end{array}$ & $\begin{array}{l}D \\
2.5 \\
0.5\end{array}$ & $\begin{array}{l}H \\
55 \\
25.8\end{array}$ & $\begin{array}{l}D \\
2.8 \\
1.2\end{array}$ & $\begin{array}{l}H \\
88.5 \\
31.8\end{array}$ & $\begin{array}{l}D \\
1.5 \\
0.4\end{array}$ \\
\hline
\end{tabular}

$H$-height of reflection in kilometers above the bottom of the layer.

$D$-distance in kilometers of reflection point from the vertical plane containing the ray incident on the layer.

The first case listed $\left(f_{0}=7.5 \mathrm{Mc}\right)$ is one which cannot arise in practice; i.e., reflection of the oblique ray from the level of maximum ion density. For the flat earth, this corresponds to an infinitely great transmission distance. But it is notable that even here, the deviation of the obliquely incident ray is probably negligible $(2.5 \mathrm{~km}$ computed), although the deviation of the corresponding vertically incident ray is not. The second case is of greater interest from a practical standpoint (reflection well below the level of maximum ionization), and here it is seen that neither ray (vertical or oblique) is deviated far from the plane of incidence. Although, in a distance of about $4 \mathrm{~km}$ (difference in computed deviations for the vertical and oblique rays at reflection), disturbing turbulence or gradients may well exist, these same disturbing elements could seriously affect the ray paths even if the effects of the magnetic field were neglected. The fact that the ionosphere is not uniform, i.e., not horizontally, or spherically stratified (the oblique ray "samples", this nonuniform medium over a relatively great distance), appears to be more pertinent to an explanation of the disagreement (or the variations in the amount of disagreement) between calculated and observed MUF than the magnetoionic deviation.

Interest in the magnetoionic deviation is then, more-or-less, academic, but it may be worthwhile to summarize the results of computations based on Booker's method. For east-west propagation, as the penetration into a given layer increases, either because of decreasing angle of incidence or increasing frequency, the amount of magnetoionic deviation increases. The extraordinary ray is deviated less than the ordinary at all latitudes and for all angles of incidence (except at the equator where neither ray is deviated out of the plane of incidence). For north-south propagation (in the vertical plane containing the magnetic field direction) no magnetoionic deviation out of this plane is experienced, but the path in the ionosphere is asymmetric, reflection points being toward the pole (ordinary) or toward the equator (extraordinary) from the path midpoint by distances of the same order as the lateral deviations suffered over an east-west path. Also, for north-south propagation the ordinary ray is reflected 
from a higher level and the extraordinary from a lower level than is the case for an east-west path. This effect, although probably relatively unimportant, should be kept in mind when comparisons are made between north-south and east-west paths.

The possible error in the transmission curve $k$ factor was discussed by Wieder, who concluded that the average discrepancy of 3 percent was unlikely to be due to the $k$ error. This conclusion is, however, somewhat uncertain and it is of interest to consider this point a little further. The $k$ factor depends in a complicated way on the electron distribution in the ionosphere and varies, to some extent, with the height of reflection and angle of incidence. The $k$ is, therefore, not constant for a given distance of transmission as was assumed in the theory underlying the transmission curves. The variation of the ratio $\left(f / f^{\prime}\right)$ with virtual height of reflection is shown in figure 26 , where $f^{\prime}$ is the vertical-incidence frequency equivalent to a frequency $f$ at oblique incidence. This figure, due to Wieder, shows that for low virtual heights of reflection the empirical values of $f / f^{\prime}$ are larger than sec $\Phi_{0}$, while at high virtual heights this effect tends to diminish. It will be noticed that the high values of virtual heights were obtained during the summer and are brought about by group retardation in the lower layers. They do not, therefore, represent the true heights of reflection.

One of the assumptions on which the transmission curves are based is that there are no horizontal gradients of electron density in the ionosphere. There is now considerable evidence for both large scale and small scale irregularities in the ionosphere, the large-scale gradients being known as tilts. Wieder, in discussing the effect of a north-south gradient on the calculation of MUF's, pointed out that "the northward deviated vertical-incidence ordinary wave encounters systematically lower densities than the obliquely incidence wave." We have seen above, however, that the lateral deviation on the equivalent vertical-incidence frequency is only about $4 \mathrm{~km}$ greater than the lateral deviation of the oblique ray at an angle of incidence of $60^{\circ}$. Hence, it appears unlikely that this would explain the observed discrepancy.

It has also been suggested that tilts might account for the discrepancy, but if the presence of a tilt shifts the point of reflection of the obliquely incident ray, then it should also produce a corresponding shift in the point of reflection of the vertically incident ray on the equivalent frequency.

Another point of considerable theoretical importance is the origin of the so-called "MUF extension". D. K. Bailey has suggested that the extension is the result of a modification in the angle of incidence of the ray on the $F$ layer due to scattering of energy by irregularities in the $E$ region. Such a mechanism would account for signals on frequencies above the classical MUF as would reflection from a rough $F$ layer. One might expect some correlation with sporadic $E$ on this hypothesis; so far very little correlation has been found.

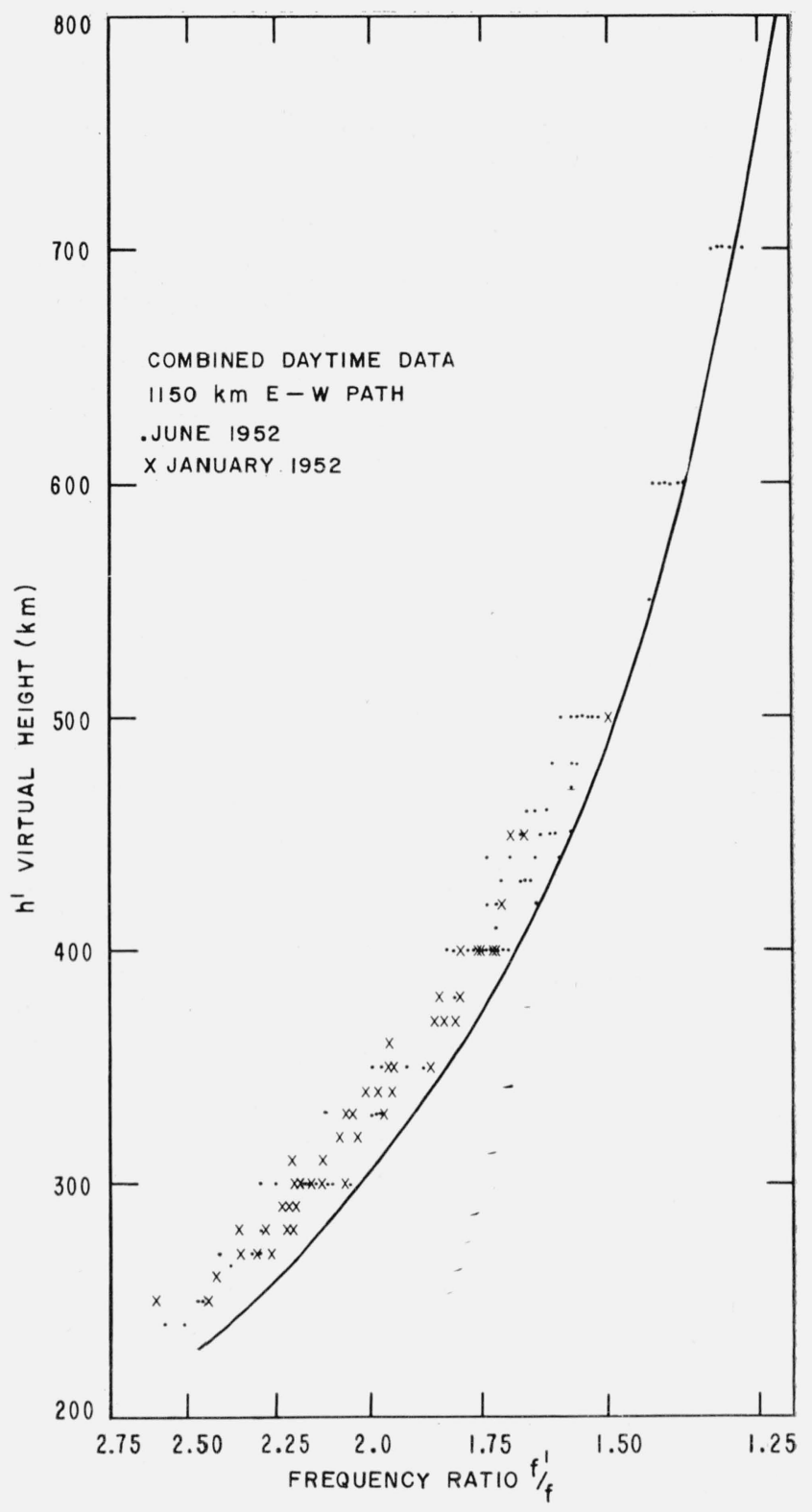

Figure 26. Graph illustrating the variation of the observed ratio of $f^{\prime} / f$ with equivalent height over the Sterling to St. Louis path.

$f^{\prime} \equiv$ the vertical-incidence frequency equivalent to the frequency $f$. The full line is the sec $\Phi_{0}$ line.

\section{Conclusions}

The results of the oblique-incidence experiments at the Bureau are in general agreement with those made by other laboratories. In particular, the discrepancy of about 3 percent between the observed and calculated MUF is similar to that found elsewhere, except that no marked percentage increase appears to occur with increase of distance as reported by the Japanese [16]. The latter probably refer to 
the maximum observed frequency, whereas [the former refer to the classical MUF. The relative importance of the high-angle ray during summer has been confirmed in nearly all the other published work on the subject.

\section{References}

[1] F. '1. Farmer and J. A. Ratcliffe, Wireless waves reflected from the ionosphere at oblique incidence, Proc. Phys. Soc. (London) 48, 839 (1936).

[2] F. T. Farmer, C. B. Childs, and A. Cowie, Critical frequency measurements of wireless waves reflected obliquely from the ionosphere, Proc. Phys. Soc. (London) 50, 767 (1938).

[3] T. L. Eckersley, S. Falloon, F. T. Farmer, and W. O. Agar, Wireless propagation and the reciprocity law, Nature 145, $222(1940)$.

[4] W. J. G. Beynon, Propagation of radio waves, Wireless Eng. 25, $322(1948)$

[5] W. Dieminger, K. H. Giesweid, and H. G. Möller, Echolotungen der ionosphäre mit veränderlicher frequenz bei schragem Einfall, NTZ-Nachr tech. Z. 8, 578 (1955).

[6] W. Dieminger and H. G. Möller, Echo sounding experiments with variable frequency at oblique incidence, Nuovo cimento [10] 4; Suppl. No. 4 (1956).

[7] H. G. Möller, Sweep-frequency oblique-incidence experiments over a distance of $1320 \mathrm{~km}$, J. atmospheric and Terrest. Phys. 9, 155 (1956).

[8] H. G. Möller, Further results of sweep frequency obliqueincidence experiments, J. Atmospheric and Terrest. Phys. 13, 173 (1958).

[9] W. Dieminger, H. G. Möller, and G. Rose, Long distance single $F$-hop transmissions, J. Atmospheric and Terrest. Phys. 13, 191 (1958).

[10] J. W. Cox and Kenneth Davies, Oblique incidence pulse transmission, Wireless Eng. 32, 35 (1955).
[11] J. H. Chapman, Kenneth Davies, and C. A. Littlewood, Radio observations of the ionosphere at oblique incidence, Can. J. Phys. 33, 713 (1955).

[12] E. Warren and E. L. Hagg, Single-hop propagation of radio waves to a distance of $5300 \mathrm{~km}$, Nature $\mathbf{1 8 1}$, 34 (1958).

[13] P. G. Sulzer and E. E. Ferguson, Sweep-frequency oblique-incidence ionospheric measurements over an $1150-\mathrm{km}$ path, Proc. I. R. E. 40, 1124 (1952).

[14] B. Wieder, Some results of a sweep-frequency propagation experiment over an $1150-\mathrm{km}$ east-west path, J. Geophys. Research 60, 395 (1955).

[15] P. G. Sulzer, Sweep-frequency pulse-transmission measurements over a 2,400-km path, J. Geophys. Research 60, 411 (1955).

[16] Unpublished, see C.C.I.R. Doc VI/53E (June 1958).

[17] F. Kift, Single-hop propagation of radio waves to a distance of $5,300 \mathrm{~km}$, Nature 181, 1459 (1958).

[18] Ionospheric radio propagation, ch 6, NBS Circ. 462 (1948).

[19] E. V. Appleton and W. J. G. Beynon, The application of ionospheric data to radio communication problems, Part I, Proc. Phys. Soc. (London ) 52, 518 (1940).

[20] N. Smith, The relation of radio sky-wave transmission to ionospheric measurements, Proc. IRE 27, 332 (1939).

[21] J. W. Wright, R. W. Knecht, and K. Davies, Ann. IGY III, Pt. I (1957).

[22] R. Silberstein, Sweep-frequency backscatter-some observations and deductions, IRE Trans. AP-2, 56 (1954).

[23] R. Silberstein, The use of sweep-frequency backscatter data for determining oblique-incidence ionospheric characteristics, J. Geophys. Research 63, 335 (1958).

[24] H. G. Booker, Application of the magneto-ionic theory to radio waves incident obliquely upon a horizontally stratified ionosphere, J. Geophys. Research 54, 243 (1949).

Boulder, Colo.

(Paper 63D2-15) 